

\section{NOTICE}

This report was prepared as an account of work sponsored by an agency of the United States Government. Neither the United States Government nor any agency thereof, nor any of their employees, makes any warranty, express or implied, or assumes any legal liability or responsibility for the accuracy, completeness, or usefulness of any information, apparatus, product, or process disclosed, or represents that its use would not infringe privately owned rights. Reference herein to any specific commercial produce, process, or service by trade name, trademark, manufacturer, or otherwise, does not necessarily constitute or imply its endorsement, recommendation, or favoring by the United States Government or any agency thereof. The views and opinions of authors expressed herein do not necessarily state or reflect those of the United States Government or any agency thereof.

\section{NOTICE}

This report has been reproduced directly from the best available copy.

Available to DOE and DOE contractors from the:

Office of Scientific and Technical Information

P.O. Box 62

Oak Ridge, TN 37831 ;

Prices available from (615) 576-8401.

Available to the public from the:

National Technical Information Service

U.S. Department of Commerce 5285 Port Royal Road

Springfield, Virginia 22161

703-487-4650 


\title{
Gyrokinetic Analysis of Ion Temperature Gradient Modes in the Presence of Sheared Flows
}

\author{
M. Artun and W. M. Tang \\ Plasma Physics Laboratory, Princeton University \\ Princeton, NJ 08543
}

\begin{abstract}
The linearized gyrokinetic equation governing electrostatic microinstabilities in the presence of sheared equilibrium flows in both the $\hat{\mathbf{z}}$ and $\hat{\mathbf{y}}$ directions has been systematically derived for a sheared slab geometry, where in the large aspect ratio limit $\hat{\mathbf{z}}$ and $\hat{\mathbf{y}}$ directions correspond to the toroidal and poloidal directions respectively. In the familiar long perpendicular wavelength regime $\left(k_{\perp} \rho_{i}<1\right)$, the analysis leads to a comprehensive kinetic differential eigenmode equation which is solved numerically. The nunierical results have been successfully cross-checked against analytic estimates in the fluid limit. For typical conditions, the Ion Temperature Gradient $\left(\eta_{i}\right)$ modes are found to be stabilized for $\hat{y}$-direction flows with a velocity shear scale comparable to that of the ion temperature gradient and velocities of a few percent of the sound speed. Sheared flows in the $\hat{z}$-direction taken alone are usually destabilizing, with the effect being independent of the sign of the flow. However, when both types are simultaneously considered, it is found that in the presence of sheared $\hat{z}$-direction flow, sheared $\hat{\mathbf{y}}$-direction flow can be either stabilizing or destabilizing depending on the relative sign of these flows. However, for sufficiently large values of $v_{y}^{\prime}$ the mode is completely stabilized regardless of the sign of
\end{abstract}


$v_{z}^{\prime} v_{y}^{\prime}$. The importance of a proper kinetic treatment of this problem is supported by comparisons with fluid estimates. In particular, when such effects are favorable, significantly smaller values of sheared $\hat{\mathbf{y}}$-direction flow are required for stability than fluid estimates would indicate.

\section{Introduction}

Investigation of the influence of sheared equilibrium flows on the confinement properties of tokamak plasmas has been a topic of much current interest. This is motivated by the fact that significant levels of radially sheared toroidal flows have been measured in neutral-beam-heated discharges. The presence of poloidal flows is supported by observations of large radial electric fields in the edge region of tokamaks. Since low frequency drift-type microinstabilities have continued to be leading candidates to account for much of the anomalous transport found in toroidally confined plasmas, it is clearly important to realistically assess how they might be affected by the combined influence of toroidal and poloidal equilibrium velocity shear. In order to provide a proper theoretical foundation for a systematic kinetic analysis of this problem, we begin by presenting a rigorous derivation of the linearized electrostatic gyrokinetic equations in the presence of sheared flows. The results are then applied to the investigation of velocity shear on the ion temperature gradient driven class of drift-type microinstabilities for a sheared slab geometry in the familiar long perpendicular wavelength regime $\left(k_{\perp} \rho_{i}<1\right){ }^{1}$

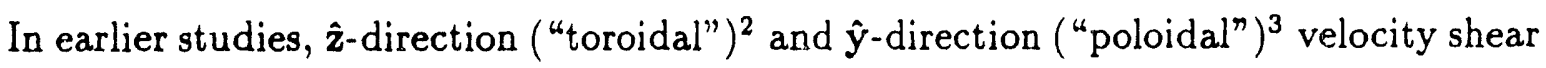
effects on ITG (ion temperature gradient) modes were separately analyzed in the fluid limit for a sheared slab geometry. The general trends found in these calculations were that the $\hat{\mathbf{z}}$-direction flows tended to be destabilizing while the $\hat{\mathbf{y}}$-direction flows could be strongly stabilizing for sufficiently large values of the flow parameter $v_{y}^{\prime}$. Results from recent kinetic analysis ${ }^{4}$ of the $\hat{y}$-direction flow problem basically support the findings from 
the fluid estimates.

The focus in the present paper is on a rigorous kinetic investigation of the combined influence of sheared flows (in the $\hat{\mathbf{z}}$ and $\hat{\mathbf{y}}$ directions) on the ITG instabilities. As in the earlier studies, ${ }^{3,4}$ it is found that for typical conditions, sheared $\hat{y}$-direction flows have a favorable influence on the ITG instabilities. More specifically, sheared flows in the $\hat{y}$ direction with a scale comparable to that of the ion temperature gradient and velocities of a few percent of the acoustic speed can stabilize these modes. When sheared flows in the $\hat{\mathbf{z}}$-direction are simultaneously taken into account, our calculations indicate that $\hat{\mathbf{y}}$ direction sheared flows can be either stabilizing or destabilizing depending on the relative sign of the flow parameters $v_{y}^{\prime}$ and $v_{z}^{\prime}$. However, for sufficiently large values of $v_{y}^{\prime}$ the mode is completely stabilized regardless of the sign of $v_{z}^{\prime} v_{y}^{\prime}$. Comparisons with results from fluid estimates are made to illustrate the importance of a kinetic treatment of this problem. In particular, when such effects are favorable, the magnitude of the sheared $\hat{y}$-direction flow required for stability is found to be significantly smaller than fluid estimates would indicate.

The remainder of this paper is organized as follows. Section II deals with a systematic derivation of the linearized gyrokinetic equations in the presence of sheared flows. The coordinate transformation and guiding center formulation used to facilitate this analysis is explained in Sec. II.A. After specifying the form of the equilibrium distribution in Sec. II.B, the derivation of the gyrokinetic equation determining the perturbed distribution function $(\delta f)$ is presented in Sec. II.C. In Sec. II.D, the basic kinetic eigenmode equation governing ITG modes in a sheared slab geometry in the presence of sheared flows is obtained by using $\delta f$ to construct the perturbed ion density response together with the usual Boltzmann response for electrons. Results from the analysis of the eigenmode equation are given in Sec. III. In Sec. III.A, analytic estimates of the velocity shear effects on ITG modes are presented, and in Sec. III.B, numerical results from the investigation 
of normal, flat, and inverted density profile cases are summarized. Finally, in Sec. IV the principal findings from the present studies and their implications are discussed.

\section{Derivation of Gyrokinetic Equations with Sheared Flows}

To properly specify the perturbed distibution function in the presence of sheared equilibrium flows, it is convenient to make a coordinate transformation from the lab frame $(\mathbf{x})$ to the rotating frame $\left(\mathbf{x}^{\prime}\right)$. We assume that for the equilibrium distribution function $F$ the flow in the lab frame is given by:

$$
\mathbf{V}_{0}(\mathbf{x})=\int \mathrm{d} \mathbf{v} \mathbf{v} F(\mathbf{x})
$$

with its the Taylor expansion around the rational surface given by $\mathbf{V}_{0}(\mathbf{x})=\mathbf{u}_{0}+\mathbf{v}_{0}(\mathbf{x})$. Here $\mathbf{u}_{0}$ is the constant part of the flow, and $\mathbf{v}_{0}(\mathbf{x})$ is the $\mathbf{x}$-dependent part. This models the radially varying flows in the toroidal geometry. Results from experimental measurements $^{5}$ as well as from theoretical calculations of neoclassical equilibrium flows ${ }^{6}$ have indicated that sheared flows in toroidal geometry can be represented as a function of the flux surface. More specifically, for a large aspect ratio tokamak, equilibrium flows can simply be approximated as a function of the radial variable only.

In the present model the sheared flows are taken to be perpendicular to the "radial" (i.e., $x$ ) direction. This choice is again consistent with expectations from theoretical calculations in a toroidal geometry and from tokamak experimental results. If all equilibrium quantities (i.e., $n_{e}, n_{i}, T_{e}, T_{i}, \mathbf{B}$ ) have a variation in the $\hat{\mathbf{x}}$-direction only, then the constant part of the sheared flow $u_{0}$ can be absorbed in the eigenfrequency in the form of a Doppler-shift term in a simple Galilean transformation and is thereby eliminated from the problem. However, a Galilean transformation for the $x$-dependent part of sheared flow $\mathrm{v}_{0}(x)$ would further complicate rather than simplify the analysis. Details of these considerations are presented in Sec. II.A. 


\section{A. Coordinate Transformation and the Guiding Center For- mulation}

The collisionless Vlasov equation analyzed is given by

$$
\frac{\mathbf{d} f}{\mathbf{d} t}=\frac{\partial f}{\partial t}+\mathbf{v} \cdot \frac{\partial f}{\partial \mathbf{x}}+\frac{q}{m}\left(\mathbf{E}+\frac{\mathbf{v} \times \mathbf{B}}{c}\right) \cdot \frac{\partial f}{\partial \mathbf{v}}=0
$$

To leading order in the Taylor expansion around the rational surface $x=0$, we take $\mathbf{V}_{0}(\mathbf{x})=\mathbf{u}_{0}+\mathrm{v}_{0}(\mathbf{x})$ with $\mathbf{V}_{0}(\mathbf{x})$ given by the first velocity moment of the distribution function. In order to simplify the problem, we make a coordinate transformation from $(t, \mathbf{x}, \mathbf{v})$ space to $\left(t^{\prime}, \mathbf{x}^{\prime}, \mathbf{c}\right)$ space, i.e.,

$$
t=t^{\prime}, \quad \mathbf{x}=\mathbf{x}^{\prime}+\mathbf{u}_{0} t^{\prime} \quad, \quad \mathbf{v}=\mathbf{c}+\mathbf{u}_{0}+\mathbf{v}_{0}(\mathbf{x})
$$

Here we require that $\mathbf{u}_{0} \cdot \hat{\mathbf{x}}=0, \mathbf{v}_{0} \cdot \hat{\mathbf{x}}=0, \mathbf{v}_{0} \cdot \nabla_{L}=0$, and $\mathbf{u}_{0} \cdot \nabla_{L}=0$ where $\nabla_{L}$ acts on equilibrium quantities (i.e., $n_{e}, T_{e}$, etc.). The partial derivatives in the lab frame then become

$$
\frac{\partial}{\partial t}=\frac{\partial}{\partial t^{\prime}}-\mathbf{u}_{0} \cdot \frac{\partial}{\partial \mathbf{x}^{\prime}} \quad, \quad \frac{\partial}{\partial \mathbf{x}}=\frac{\partial}{\partial \mathbf{x}^{\prime}}-\nabla \mathbf{v}_{0} \cdot \frac{\partial}{\partial \mathbf{c}} \quad, \quad \frac{\partial}{\partial \mathbf{v}}=\frac{\partial}{\partial \mathbf{c}} .
$$

Hence, the Vlasov equation in the "rotating" frame is given by:

$$
\frac{\mathbf{d} f}{\mathbf{d} t}=\frac{\partial f}{\partial t^{\prime}}+\left(\mathbf{v}_{0}+\mathbf{c}\right) \cdot \frac{\partial f}{\partial \mathbf{x}^{\prime}}-\mathbf{c} \cdot \nabla \mathbf{v}_{0} \cdot \frac{\partial f}{\partial \mathbf{c}}+\frac{q}{m}\left(\mathbf{E}^{\prime}+\frac{\mathbf{c} \times \mathbf{B}}{c}\right) \cdot \frac{\partial f}{\partial \mathbf{c}}=0
$$

where $\mathbf{E}^{\prime}=\mathbf{E}+\left[\left(\mathbf{u}_{0}+\mathbf{v}_{0}\right) \times \mathbf{B}\right] / c$. Here the perpendicular electric field necessary to set up an $\mathbf{E} \times \mathbf{B}$ drift velocity in the "poloidal" direction ( $y$-direction) is given by

$$
\mathbf{E}_{0}(\mathbf{x})=-\frac{\left(\mathbf{u}_{0}+\mathrm{v}_{0}\right) \times \mathbf{B}}{c} .
$$

Note that $\mathbf{E}=\mathbf{E}^{\prime}+\mathbf{E}_{0}$ with $\mathbf{E}^{\prime}$ being the electric field observed in the moving frame.

We next proceed with the transformation to the guiding center frame. Specifically, the familiar ${ }^{7-10}$ guiding center coordinate transformation from $\left(t^{\prime}, \mathbf{x}^{\prime}, \mathbf{c}\right)$ to $\left(t^{\prime}, \mathbf{R}, E, \mu, \phi\right)$ with 
$\mathbf{R}=\mathbf{x}^{\prime}+(\mathbf{c} \times \hat{\mathbf{b}}) / \Omega_{c}, \mu=c_{\perp}^{2} / 2 B, E=c^{2} / 2, \Omega_{c}=(q B / m c)$, and $\mathbf{c}=c_{\|} \hat{\mathbf{b}}+c_{\perp}\left(\cos \phi \hat{\mathbf{e}}_{1}+\right.$ $\left.\sin \phi \hat{\mathbf{e}}_{2}\right)$ is applied to Eq. (5). The spatial derivative becomes

$$
\frac{\partial}{\partial \mathbf{x}^{\prime}}=\frac{\partial}{\partial \mathbf{R}}+\frac{\partial \mu}{\partial \mathbf{x}^{\prime}} \frac{\partial}{\partial \mu}+\frac{\partial \phi}{\partial \mathbf{x}^{\prime}} \frac{\partial}{\partial \phi}-\frac{\partial\left(\hat{\mathbf{b}} / \Omega_{c}\right)}{\partial \mathbf{x}^{\prime}} \times \mathbf{c} \cdot \frac{\partial}{\partial \mathbf{R}}
$$

with

$$
\begin{aligned}
& \frac{\partial \mu}{\partial \mathbf{x}^{\prime}}=-\frac{1}{B}\left(\mu \frac{\partial B}{\partial \mathbf{x}^{\prime}}+c_{\|} \frac{\partial \hat{\mathbf{b}}}{\partial \mathbf{x}^{\prime}} \cdot \mathbf{c}_{\perp}\right) \\
& \frac{\partial \phi}{\partial \mathbf{x}^{\prime}}=\frac{\partial \hat{\mathbf{e}}_{2}}{\partial \mathbf{x}^{\prime}} \cdot \hat{\mathbf{e}}_{1}+\frac{c_{\|}}{c_{\perp}} \frac{\partial \hat{\mathbf{b}}}{\partial \mathbf{x}^{\prime}} \cdot(\mathbf{c} \times \hat{\mathbf{b}}),
\end{aligned}
$$

and using

$$
\frac{\partial E}{\partial \mathbf{c}}=\mathbf{c} \quad, \quad \frac{\partial \mu}{\partial \mathbf{c}}=\frac{\mathbf{c}_{\perp}}{B}, \quad \frac{\partial \phi}{\partial \mathbf{c}}=-\frac{\mathbf{c} \times \hat{\mathbf{b}}}{c_{\perp}^{2}},
$$

the velocity derivative can be expressed as

$$
\frac{\partial}{\partial \mathbf{c}}=\mathbf{c} \frac{\partial}{\partial E}+\frac{\mathbf{c}_{\perp}}{B} \frac{\partial}{\partial \mu}-\frac{\mathbf{c} \times \hat{\mathbf{b}}}{c_{\perp}^{2}} \frac{\partial}{\partial \phi}+\frac{\mathbf{I} \times \hat{\mathbf{b}}}{\Omega_{\mathrm{c}}} \cdot \frac{\partial}{\partial \mathbf{R}} .
$$

It is convenient to introduce the definitions

$$
\begin{aligned}
\lambda_{B_{1}} & =-\frac{\partial\left(\hat{\mathbf{b}} / \Omega_{c}\right)}{\partial \mathbf{x}^{\prime}} \times \mathbf{c} \cdot \frac{\partial}{\partial \mathbf{R}} \\
\lambda_{B_{2}} & =\frac{\partial \mu}{\partial \mathbf{x}^{\prime}} \frac{\partial}{\partial \mu}+\frac{\partial \phi}{\partial \mathbf{x}^{\prime}} \frac{\partial}{\partial \phi}
\end{aligned}
$$

The Vlasov equation in the guiding center coordinates can then be expressed as

$$
\begin{aligned}
\frac{\mathrm{d} f}{\mathrm{~d} t}= & \frac{\partial f}{\partial t^{\prime}}+\mathbf{v}_{0} \cdot \frac{\partial f}{\partial \mathbf{R}}+c_{1} \frac{\partial f}{\partial R_{\|}}+\left(\mathbf{v}_{0}+\mathbf{c}\right) \cdot\left[\lambda_{B_{1}} f+\lambda_{B_{2}} f\right] \\
& -\mathbf{c} \cdot \nabla \mathbf{v}_{0} \cdot\left[\mathbf{c} \frac{\partial f}{\partial E}+\frac{\mathbf{c}_{\perp}}{B} \frac{\partial f}{\partial \mu}-\frac{\mathbf{c} \times \hat{\mathbf{b}}}{c_{\perp}^{2}} \frac{\partial f}{\partial \phi}+\frac{\mathbf{I} \times \hat{\mathbf{b}}}{\Omega_{\mathbf{c}}} \cdot \frac{\partial f}{\partial \mathbf{R}}\right] \\
& -\Omega_{c} \frac{\partial f}{\partial \phi}
\end{aligned}
$$

Here $\nabla^{\prime} \mu$ and $\nabla^{\prime} \phi$ can be written as $\nabla \mu$ and $\nabla \phi$ since $\mathbf{B}, \hat{\mathbf{e}}_{1}$ and $\hat{\mathbf{e}}_{2}$ are functions of $\mathbf{x}$ only with no dependence on $v$. 


\section{B. Equilibrium Distribution}

In a slab geometry with the equilibrium magnetic field given by $\mathbf{B}=\hat{\mathbf{z}}+\left(x / L_{s}\right) \hat{\mathbf{y}}$, it is customary to associate $\hat{\mathbf{z}}, \hat{\mathbf{y}}$, and $\hat{\mathbf{x}}$ with the toroidal, poloidal and radial directions of a large aspect ratio tokamak respectively. For drift instabilities the appropriate ordering using $\epsilon$ as the smallness parameter is $\omega \sim k_{\|} v_{t h_{i}},\left(\omega / \Omega_{c i}\right) \sim \mathrm{O}(\epsilon), \rho_{i} / L_{n} \sim \rho_{i} / L_{T_{i}} \sim \epsilon$ and $k_{\perp} \rho_{i} \sim O(1)$, where $L_{n}$ and $L_{T_{i}}$ are density and temperature gradient scale lengths respectively. Denoting the equilibrium distribution function by $F=F_{0}+F_{1}+\mathrm{O}\left(\epsilon^{2}\right)$, the leading order term of the Vlasov operator in the guiding center coordinates is $-\Omega_{c} \partial F / \partial \phi$. Hence, to this lowest order, we have $F_{0}=F_{0}(\mathbf{R}, E, \mu)$. If $F_{0}$ is isotropic, then $F_{0}=$ $F_{0}(\mathbf{R}, E)$. To next order we get

$$
c_{\|} \frac{\partial F_{0}}{\partial R_{\|}}-\mathbf{c} \cdot \nabla \mathbf{v}_{0} \cdot \mathbf{c} \frac{\partial F_{0}}{\partial E}+\mathbf{v}_{0} \cdot \frac{\partial F_{0}}{\partial \mathbf{R}}-\Omega_{c} \frac{\partial F_{1}}{\partial \phi}=0
$$

The gyrophase average of this equation yields

$$
\left\langle\mathbf{c} \cdot \nabla \mathbf{v}_{0} \cdot \mathbf{c}\right\rangle_{\phi}=\langle\mathbf{c c}\rangle_{\phi}: \nabla \mathbf{v}_{0}=c_{\|}^{2} \hat{\mathbf{b}} \hat{\mathbf{b}}+\left(c_{\perp}^{2} / 2\right)(\mathbf{I}-\hat{\mathbf{b}} \hat{\mathbf{b}}): \nabla \mathbf{v}_{0}
$$

and, since $\hat{\mathrm{b}} \cdot \nabla \mathrm{v}_{0}=0$ and $\mathbf{I}: \nabla \mathrm{v}_{s}=\nabla \cdot \mathrm{v}_{0}=0$, we obtain

$$
c_{\|} \frac{\partial F_{0}}{\partial R_{\|}}+v_{0} \cdot \frac{\partial F_{0}}{\partial \mathbf{R}}=0
$$

This result indicates that $F_{0}=F_{0}(E, X)$, since both $\hat{\mathrm{b}}$ and $\mathrm{v}_{0}$ lie in the $y z$-plane, where $X=\hat{\mathbf{x}} \cdot \mathbf{R}$.

With respect to $F_{1}$, we find

$$
-\mathbf{c} \cdot \nabla \mathbf{v}_{0} \cdot \mathbf{c} \frac{\partial F_{0}}{\partial E}=\Omega_{c} \frac{\partial F_{1}}{\partial \phi}
$$

Denoting $F_{1}=\tilde{F}_{1}+\left\langle F_{1}\right\rangle_{\phi}$, with $2 \pi\langle\cdots\rangle_{\phi}=\int_{0}^{2 \pi} \mathrm{d} \phi \cdots$, we get

$$
\tilde{F}_{1}=-\frac{1}{\Omega_{c}}\left[\int \operatorname{cc} d \phi\right]: \nabla \mathrm{v}_{0} \frac{\partial F_{0}}{\partial E}
$$


For the slab geometry the rather complicated term, $\int \operatorname{cc} d \phi$, can be considerably simplified. Without loss of generality, we may take $\hat{\mathbf{e}}_{1}=\hat{\mathbf{x}}$ and $\hat{\mathbf{e}}_{2}=\hat{\mathbf{y}}-\left(x / L_{s}\right) \hat{\mathbf{z}}$ with $\hat{\mathbf{e}}_{1} \times \hat{\mathbf{e}}_{2}=\hat{\mathrm{b}}$. Thus, given that $\hat{\mathbf{x}} \cdot \mathrm{v}_{0}=0, \hat{\mathrm{b}} \cdot \nabla \mathrm{v}_{0}=0$ and $\mathrm{v}_{0} \cdot \nabla \mathrm{v}_{0}=0$, only the $\hat{\mathrm{b}} \hat{\mathbf{e}}_{1}$ and $\hat{\mathbf{e}}_{2} \hat{\mathrm{e}}_{1}$ components of the dyadic are of interest here. Hence, the first order correction to the equilibrium function reduces to

$$
\tilde{F}_{1}=-\frac{1}{\Omega_{c}}\left(c_{\perp} c_{\| !} \sin \phi \hat{\mathbf{e}}_{1} \hat{\mathbf{b}}+c_{\perp}^{2} \frac{\sin ^{2} \phi}{2} \hat{\mathbf{e}}_{1} \hat{\mathbf{e}}_{2}\right): \nabla \mathrm{v}_{0} \frac{\partial F_{0}}{\partial E}
$$

\section{Calculation of the Perturbed Distribution Function}

The unperturbed Vlasov operator in the coordinate frame $\left(t^{\prime}, \mathbf{x}^{\prime}, \mathbf{c}\right)$ specified by Eq. (3) can be written as

$$
\mathcal{L}=\frac{\partial}{\partial t^{\prime}}+\left(\mathrm{v}_{0}+\mathrm{c}\right) \cdot \frac{\partial}{\partial \mathbf{x}_{\mathrm{c}}}-\left(\mathrm{v}_{0}+\mathrm{c}\right) \cdot \nabla \mathrm{v}_{0} \cdot \frac{\partial}{\partial \mathbf{c}}+\frac{q}{m}\left(\frac{\mathrm{c} \times \mathbf{B}}{c}\right) \cdot \frac{\partial}{\partial \mathbf{c}}
$$

In guiding center coordinates it has the form

$$
\begin{aligned}
\mathcal{L}_{g}= & \frac{\partial}{\partial t^{\prime}}+\mathrm{v}_{0} \cdot \frac{\partial}{\partial \mathbf{R}}+c_{\|} \frac{\partial}{\partial R_{\|}}+\left(\mathrm{v}_{0}+\mathbf{c}\right) \cdot\left[\lambda_{B_{1}}+\lambda_{B_{2}}\right]-\Omega_{c} \frac{\partial}{\partial \phi} \\
& -\mathbf{c} \cdot \nabla \mathrm{v}_{0} \cdot\left[\mathbf{c} \frac{\partial}{\partial E}+\frac{\mathbf{c}_{\perp}}{B} \frac{\partial}{\partial \mu}-\frac{\mathbf{c} \times \hat{\mathbf{b}}}{c_{\perp}^{2}} \frac{\partial}{\partial \phi}+\frac{\mathbf{I} \times \hat{\mathbf{b}}}{\Omega_{c}} \cdot \frac{\partial}{\partial \mathbf{R}}\right]
\end{aligned}
$$

For electrostatic perturbations, $E^{\prime}=-\nabla \Phi$, and the perturbed Vlasov equation to order $\epsilon$ is given by

$$
\mathcal{L}[\hat{c} f]-\frac{q}{m} \nabla \Phi \cdot \frac{\partial F}{\partial \mathbf{c}}=0
$$

where $F$ is the equilibrium distribution function that vanishes under the unperturbed Vlasov operator. We follow the usual procedure ${ }^{7}$ and separate $\delta f$ into adiabatic and non-adiabatic parts; i.e.,

$$
\delta f=-\frac{q}{T(0)} \Phi F+g
$$


Letting $\mathcal{L}$ act on the adiabatic part, (i.e., $-(q / T) \Phi F)$ and $\mathcal{L}_{g}$ act on $g$, we obtain

$$
\mathcal{L}\left[\frac{q}{T(0)} \Phi F\right]=\frac{q}{T(0)} \frac{\partial \Phi}{\partial t^{\prime}} F+\left(\mathbf{v}_{0}+\mathbf{c}\right) \cdot \frac{\partial \Phi}{\partial \mathbf{x}^{\prime}} \frac{q}{T(0)} F .
$$

The perturbed Vlasov equation can be then written as

$$
\begin{aligned}
\frac{\mathrm{d} \delta f}{\mathrm{~d} t}= & -\frac{q}{T(0)} \frac{\partial \Phi}{\partial t^{\prime}}\left(F_{0}+F_{1}\right)-\left(\mathbf{v}_{0}+\mathbf{c}\right) \cdot \frac{\partial \Phi}{\partial \mathbf{x}^{\prime}} \frac{q}{T(0)}\left(F_{0}+F_{1}\right) \\
& +\frac{\partial g}{\partial t^{\prime}}+\mathbf{v}_{0} \cdot \frac{\partial g}{\partial \mathbf{R}}+c_{\|} \frac{\partial g}{\partial R_{\|}}+\left(\mathbf{v}_{0}+\mathbf{c}\right) \cdot\left[\lambda_{B_{1}}+\lambda_{B_{2}}\right] g \\
& -\mathbf{c} \cdot \nabla \mathbf{v}_{0} \cdot\left[\mathbf{c} \frac{\partial g}{\partial E}+\frac{\mathbf{c}_{\perp}}{B} \frac{\partial g}{\partial \mu}-\frac{\mathbf{c} \times \hat{\mathbf{b}}}{c_{\perp}^{2}} \frac{\partial g}{\partial \phi}+\frac{\mathbf{I} \times \hat{\mathbf{b}}}{\Omega_{c}} \cdot \frac{\partial g}{\partial \mathbf{R}}\right]-\Omega_{c} \frac{\partial g}{\partial \phi} \\
= & \frac{q}{m} \nabla \Phi \cdot\left[\mathbf{c} \frac{\partial F_{0}}{\partial E}+\frac{\mathbf{I} \times \hat{\mathbf{b}}}{\Omega_{c}} \cdot \frac{\partial F_{0}}{\partial \mathbf{R}}+\frac{\partial F_{1}}{\partial \mathbf{c}}\right]
\end{aligned}
$$

The lowest order term in Eq. (24) is

$$
-\Omega_{c} \frac{\partial g}{\partial \phi}=0
$$

such that $\langle g\rangle_{\phi}=g$. After taking the gyrophase average of Eq. (24), we observe that

$$
\left\langle\nabla \Phi \cdot \frac{\partial\left\langle F_{1}\right\rangle_{\phi}}{\partial \mathbf{c}}\right\rangle_{\phi} \sim O\left(\epsilon^{2}\right)
$$

For the gyrophase dependent part of $F_{1}$, (i.e., $\tilde{F}_{1}$ ) we find

$$
\frac{\partial \tilde{F}_{1}}{\partial \mathbf{c}}=-\frac{m}{T} \mathbf{c} \tilde{F}_{1}+\frac{1}{\Omega_{c}} \frac{m}{T}\left(c_{\|} \hat{\mathbf{e}}_{2} \hat{\mathbf{e}}_{1} \hat{\mathbf{b}}+c_{\perp} \sin \phi \hat{\mathbf{e}}_{2} \hat{\mathbf{e}}_{1} \hat{\mathbf{e}}_{2}\right): \nabla \mathbf{v}_{0} F_{0}
$$

Since terms like $F_{1}\left(\partial \Phi / \partial t^{\prime}\right)$ and $\mathbf{v}_{0} \cdot \nabla \Phi F_{1}$ are $O\left(\epsilon^{2}\right)$, gyroaveraging the perturbed Vlasov equation to $O(\epsilon)$ leads to the result

$$
\langle\mathcal{L}[\delta f]\rangle_{\phi}=-\frac{q}{T} J_{0}\left(\frac{k_{\perp} c_{\perp}}{\Omega_{c}}\right) \frac{\partial \Phi_{G}}{\partial t^{\prime}} F_{0}-\frac{q}{T} J_{0}\left(\frac{k_{\perp} c_{\perp}}{\Omega_{c}}\right) \mathbf{v}_{0} \cdot \frac{\partial \Phi_{G}}{\partial \mathbf{R}} F_{0}
$$




$$
\begin{aligned}
& +\frac{\partial g}{\partial t^{\prime}}+\mathrm{v}_{0} \cdot \frac{\partial g}{\partial \mathbf{R}}+c_{\|} \frac{\partial g}{\partial R_{\|}}=\frac{q}{m} \frac{\partial \Phi_{G}}{\partial \mathbf{R}} \times \frac{\hat{\mathbf{b}}}{\Omega_{c}} \cdot \frac{\partial F_{0}}{\partial \mathbf{R}} J_{0}\left(\frac{k_{\perp} c_{\perp}}{\Omega_{c}}\right) \\
& +\frac{q}{T} J_{0}\left(\frac{k_{\perp} c_{\perp}}{\Omega_{c}}\right) \hat{\mathbf{e}}_{2} \cdot \frac{\partial \Phi_{G}}{\partial \mathbf{R}} \hat{\mathbf{e}}_{1} \cdot \nabla \mathbf{v}_{0} \cdot \hat{\mathbf{b}} c_{\|} F_{0}
\end{aligned}
$$

where $\Phi_{G}$ is the perturbed potential at the guiding center ${ }^{8}$ and $\Phi \simeq \Phi_{G} \exp (i L)$ with $L=\mathbf{k} \cdot \boldsymbol{\rho}$ and $\rho$ identified as the gyroradius. Here we have also made use of the fact that for a sheared slab geometry,

$$
\left\langle\left(\mathbf{v}_{0}+\mathbf{c}\right) \cdot\left(\lambda_{B_{1}}+\nabla \mu \frac{\partial}{\partial \mu}\right)\right\rangle_{\phi} \simeq O\left(\epsilon^{2}\right) .
$$

Thus, the final form of the electrostatic gyrokinetic equation with sheared flows is given by

$$
\begin{aligned}
\frac{\partial g}{\partial t^{\prime}}+\mathbf{v}_{0} \cdot \frac{\partial g}{\partial \mathbf{R}}+c_{\|} \frac{\partial g}{\partial R_{l i}}= & \frac{q}{T} J_{0} F_{0}\left(\frac{\partial \Phi_{G}}{\partial t^{\prime}}+\mathbf{v}_{0} \cdot \frac{\partial \Phi_{G}}{\partial \mathbf{R}}\right) \\
& +\frac{q}{m} J_{0} \frac{\partial \Phi_{G}}{\partial \mathbf{R}} \times \frac{\hat{\mathbf{b}}}{\Omega_{c}} \cdot \frac{\partial F_{0}}{\partial \mathbf{R}} \\
& \frac{q}{T} J_{0} F_{0} c_{\|} \hat{\mathbf{e}}_{2} \cdot \frac{\partial \Phi_{G}}{\partial \mathbf{R}} \hat{\mathbf{e}}_{1} \cdot \nabla \mathbf{v}_{0} \cdot \hat{\mathbf{b}}
\end{aligned}
$$

where the argument of the Bessel function is $\left(k_{\perp} c_{\perp} / \Omega_{c}\right)$.

\section{Eigenmode Equation}

In proceeding with the derivation of the governing eigenmode equation, we adopt the familiar ansatz, $\Phi \sim \tilde{\Phi} \exp \left(i \mathbf{k} \cdot \mathbf{x}-i \omega^{\prime} t^{\prime}\right)$. Choosing $v_{0}(x)=\left[v_{0 y}\left(x / L_{v}\right) \hat{\mathbf{y}}+v_{0 z}\left(x / L_{v}\right) \hat{\mathbf{z}}\right]$, Eq. (30) to leading order yields

$$
\left(\mathbf{k} \cdot \mathbf{v}_{0}+k_{\|} c_{\|}-\omega^{\prime}\right) y=\frac{q}{T} J_{0} F_{0} \Phi_{G}\left(\mathbf{k} \cdot \mathbf{v}_{0}-\omega^{\prime}\right)
$$




$$
\begin{aligned}
& -\omega_{*} \frac{q}{m} J_{0} F_{0} \Phi_{G}\left(1+\eta\left(\frac{E}{T}-\frac{3}{2}\right)\right) \\
& +\omega_{*} \frac{q}{m} J_{0} F_{0} \Phi_{G}\left(\frac{L_{n}}{L_{v}} \frac{c_{\|} v_{0 z}}{(T / m)}\right) .
\end{aligned}
$$

Here $\omega^{\prime}$ is the frequency observed in the moving frame; i.e., $\omega^{\prime}=\omega-\mathbf{k} \cdot \mathbf{u}_{0}$. Defining $\omega_{*}=\left(c k_{y} T / q B L_{n}\right), \eta=L_{n} / L_{T}$ and $\tilde{\omega}=\omega^{\prime}-\mathbf{k} \cdot v_{0}$, we then obtain

$$
\delta f=-\frac{q}{\Gamma} \Phi F_{0}-\frac{q}{T} \Phi_{G} F_{0} J_{0} \frac{\tilde{\omega}+\omega_{*}\left(1+\eta(E / T-3 / 2)-\omega_{*}\left(L_{n} / L_{v}\right)\left(m c_{\|} v_{0 z} / T\right)\right)}{k_{\|} c_{\|}-\tilde{\omega}}
$$

Performing the usual velocity space integrations and recognizing that

$$
\oint \Phi_{G} d \phi=2 \pi J_{0}\left(k_{\perp} c_{\perp} / \Omega_{c}\right) \Phi
$$

we arrive at the following expression for the perturbed ion density response:

$$
\begin{aligned}
\frac{\delta n}{n_{0}}= & -\frac{q}{T} \Phi-\frac{q}{T} \Phi\left[\left(\tilde{\omega}+\omega_{*}\left(1-\frac{3}{2} \eta\right)\right) \Gamma_{0}(b) \frac{1}{\tilde{\omega}} \zeta Z(\zeta)\right. \\
& -\frac{\omega_{*}}{\tilde{\omega}} \frac{L_{n}}{L_{v}} \frac{2 v_{0 z}}{v_{t}} \zeta(1+\zeta Z(\zeta)) \Gamma_{0}(b)+\omega_{*} \eta \Gamma_{0}(b) \frac{\zeta^{2}}{\tilde{\omega}}(1+\zeta Z(\zeta)) \\
& \left.+\omega_{*} \eta\left(\Gamma_{0}(b)+b \Gamma_{1}(b)-b \Gamma_{0}(b)\right) \frac{1}{\tilde{\omega}} \zeta Z(\zeta)\right]
\end{aligned}
$$

where $\Gamma_{n}(b)=I_{n}(b) \exp (-b), b=\left(k_{\perp}^{2} v_{t}^{2} / \Omega_{c}^{2}\right), \mathrm{I}_{n}(b)$ is the modified Bessel function, and $Z(\zeta)$ denotes the plasma dispersion function with $\zeta=\left(\tilde{\omega} / k_{\|} v_{t}\right)$. Combining this result with an adiabatic electron density response in the quasineutrality equation then gives

$$
\delta n_{e}=\delta n_{i}=n_{0} \frac{e \Phi}{T_{e}}
$$

Since non-adiabatic kinetic electron effects on $\eta_{i}$-modes are knowr. to be weak ${ }^{11}$ we focus our attention on the kinetic response of the ions. In the long wavelength regime, $k_{\perp} \rho_{i}<1$,

$$
b_{i}=\rho_{i}^{2}\left(k_{x}^{2}+k_{y}^{2}\right) \simeq \rho_{i}^{2}\left(-\frac{\partial^{2}}{\partial x^{2}}+k_{y}^{2}\right)
$$


so that the basic eigenmode equation reduces to the familiar second order differential equation form ${ }^{1}$ :

$$
\frac{\partial^{2} \Phi}{\partial \bar{x}^{2}}+\left(-b_{s}+\frac{P(\bar{x})}{R(\bar{x})}\right) \Phi=0
$$

where

$$
\begin{aligned}
P(\bar{x})= & \tau\left[\tilde{\Omega}+\tilde{\Omega} \tau+\left(\tilde{\Omega} \tau+1-\frac{\eta_{i}}{2}\right) \zeta_{i} Z\left(\zeta_{i}\right)+\eta_{i} \zeta_{i}^{2}\left(1+\zeta_{i} Z\left(\zeta_{i}\right)\right)\right. \\
& \left.-2 \frac{v_{0 z}}{v_{t i}} \frac{L_{n}}{L_{v}} \zeta_{i}\left(1+\zeta_{i} Z\left(\zeta_{i}\right)\right)\right] \\
R(\bar{x})= & \left.\left(\tilde{\Omega} \tau+1+\frac{\eta_{i}}{2}\right) \zeta_{i} Z\left(\zeta_{i}\right)+\eta_{i} \zeta_{i}^{2}\left(1+\zeta_{i} Z\left(\zeta_{i}\right)\right)-2 \frac{v_{0 z}}{v_{t i}} \frac{L_{n}}{L_{v}} \zeta_{i}\left(1+\zeta_{i} Z\left(\zeta_{i}\right)\right)\right)
\end{aligned}
$$

and

$$
\begin{array}{ccc}
\omega_{* i}=\frac{c k_{y} T}{|e| B L_{n}} \quad \zeta_{i}=\frac{\tilde{\omega}}{k_{\|} v_{t}} & b=\frac{k_{\perp}^{2} v_{t i}^{2}}{\Omega_{c i}{ }^{2}} \quad \tilde{\Omega}=\frac{\tilde{\omega}}{\omega_{* e}} \\
\Omega_{c i}=\frac{e B}{m_{i} c} \quad L_{n}=\frac{\partial \ln n_{0}}{\partial x} & c_{s}=\sqrt{\frac{T_{e}}{m_{i}}} \quad \bar{x}=\frac{x}{\rho_{s}} \\
\rho_{i}=\frac{v_{t i}}{\Omega_{c i}} \quad \rho_{s}=\frac{c_{s}}{\Omega_{c i}} & \eta_{i}=\frac{\partial \ln T_{i} / \partial x}{\partial \ln n_{0} / \partial x}
\end{array}
$$

\section{Eigenmode Analysis of Ion Temperature Gradient Insta- bilities}

The eigenmode equation derived in the previous section can be solved numerically using a WKB (Wenzel-Kramer-Brillouin) shooting code. ${ }^{12}$ In the fluid limit, where we assume a large argument for the $Z$-function, (i.e., $\zeta_{i}>1$ ), the plasma dispersion function can be represented as an asymptotic series. Keeping terms of order $\zeta_{i}^{-2}$, the eigenmode equation reduces to the Weber equation ${ }^{1}$ with the corresponding eigenvalue condition for $\Omega$ in the form of a polynomial equation. To properly capture the effects of $\hat{y}$-direction 
velocity shear, terms up to order $\zeta_{i}^{-4}$ need to be kept which in turn are perturbatively incorporated in the eigenvalue condition. In the case where both $\hat{\mathbf{y}}$ and $\hat{\mathbf{z}}$-direction velocity shear are taken into account, the effects are coupled, and a perturbative analysis is unnecessary. Recall that previous studies ${ }^{3}$ have shown that the fundamental mode in the presence of only $\hat{\mathbf{y}}$-direction velocity shear can be expressed as a combination of fundamental and higher order modes in the absence of such velocity shear. However, when both $\hat{\mathbf{y}}$ and $\hat{\mathbf{z}}$ velocity shear are taken into account, the combined effect is mainly manifested in the modification of the fundamental ("ground-state") eigenfunction.

\section{A. Analytic Results: Fluid Limit}

\section{Fluid Equations: Local Analysis}

Before attempting to solve the eigenmode equation governing ITG instabilities it is appropriate to first give a simple local estimate illustrating how the mode is destabilized in the presence of parallel velocity shear. For this analysis we consider a slab geometry with $\mathbf{B}=B \hat{z} ;, k_{x}=0$, and no equilibrium electric field. The ions have a flow $V_{0 z}(x)$ in the $\hat{\mathbf{z}}$-direction, where the shear scale is similar to the temperature scale, and the velocity is comparable to the sound velocity. The ion velocity can be expressed as

$$
\mathbf{v}=V_{0 z}(x) \hat{\mathbf{z}}+v_{1 z} \hat{\mathbf{z}}+\frac{c \mathbf{E} \times \mathbf{B}}{B^{2}}
$$

where $v_{1 z}$ is the perturbed velocity, and the $\mathbf{E} \times \mathbf{B}$ flow is in the $\hat{\mathbf{x}}$-direction. The model for the flow is specified by

$$
V_{0 z}(x)=v_{0 z} \frac{x}{L_{v}} \quad \text { with } \quad v_{0 z} \sim v_{t i}
$$

with $x \ll L_{v}, V_{0 z}(x) \ll v_{t i}$. The quasineutrality condition with adiabatic electrons gives

$$
n_{e}=n_{i}=n_{0} \frac{e \Phi}{T}
$$


and the parallel momentum balance, pressure balance, and continuity equations are expressed as

$$
\begin{gathered}
m_{i} n_{0} \frac{\partial v_{1 z}}{\partial t}+m_{i} n_{0}\left[\frac{c \mathbf{E} \times \mathbf{B}}{B^{2}}\right]_{x} \frac{\partial V_{0 z}}{\partial x}=-\frac{\partial p_{i}}{\partial z}-e n_{0} \frac{\partial \Phi}{\partial z} \\
\frac{\partial p_{i}}{\partial t}+\left[\frac{c \mathbf{E} \times \mathbf{B}}{B^{2}}\right]_{x} \frac{\partial p_{0}}{\partial x}+\gamma p_{0} \frac{\partial v_{1 z}}{\partial z}=0 \\
\frac{\partial n_{i}}{\partial t}+n_{0} \frac{\partial v_{1 z}}{\partial z}=0 .
\end{gathered}
$$

Solving these equations in the usual way produces the dispersion relation,

$$
\omega\left[\frac{T_{e}}{T_{i}} \omega^{2}-\left(\frac{T_{e}}{T_{i}}+\gamma\right) k_{z}^{2} c_{s}^{2}\right]=\omega_{*} T_{e} k_{z}^{2} c_{s}^{2}-\frac{T_{e}}{T_{i}} \omega_{* V e} \omega k_{z} v_{0 z}
$$

where

$$
\omega_{* V e} \equiv \frac{c k_{y} T_{e}}{e B L_{v}} \quad, \quad \omega_{* T e} \equiv \frac{c k_{y} T_{e}}{e B L_{T_{e}}} \quad, \quad c_{s}^{2} \equiv \frac{p_{0}}{m_{i} n_{0}} \quad, \quad L_{T_{e}} \equiv-\frac{\partial \ln T_{e}}{\partial x}
$$

and $L_{T_{e}}$ is taken to be equivalent to $L_{T_{i}}$. This simplified dispersion relation illustrates the effect of parallel velocity shear on the ITG mode. There exists an unstable mode if

$$
\left[(\tau+\gamma) \Omega_{0}^{2}-\tau \Omega_{0}\left(\frac{L_{T}}{L_{v}}\right)\left(\frac{v_{0 z}}{c_{s}}\right)\right]^{3}>\frac{27}{4} \Omega_{0}^{4}
$$

where $\Omega_{0} \equiv\left(k_{z} c_{s} / \omega_{*} T_{e}\right)$. Note that the sign of the parallel velocity shear term is not important because a combination of $k_{z}$ and $k_{y}$ can always be found to give an $\Omega_{0}$ with the appropriate sign to increase the instability.

In order to demonstrate that a dispersion relation similar to the one obtained through the preceding fluid-type analysis can be obtained from the gyrokinetic equation, we consider the flat density gradient limit. In the limit $\rho_{i} \rightarrow 0$, the perturbed ion density response is given by

$$
\begin{aligned}
\frac{\delta n_{i}}{n_{0}}= & -\frac{e \Phi}{T_{i}}-\frac{e \Phi}{T_{i}}\left[\left(1-\frac{1}{2} \frac{\omega_{*} T_{i}}{\omega}\right) \zeta_{i} Z\left(\zeta_{i}\right)-2 \frac{\omega_{*} V_{i}}{\omega} \frac{v_{0 z}}{v_{t_{i}}} \zeta_{i}\left(1+\zeta_{i} Z\left(\zeta_{i}\right)\right)\right. \\
& \left.+\frac{\omega_{*} T_{i}}{\omega} \zeta_{i}^{2}\left(1+\zeta_{i} Z\left(\zeta_{i}\right)\right)\right] .
\end{aligned}
$$


Using the adiabatic electron response in the quasineutrality condition and making the usual expansions for the $Z$-function in the large argument limit then yields the dispersion relation

$$
\omega\left[\frac{T_{e}}{T_{i}} \omega^{2}-\frac{T_{e}}{T_{i}} k_{z}^{2} c_{s}^{2}\right]=\omega_{* T e} k_{z}^{2} c_{s}^{2}-\frac{T_{e}}{T_{i}} \omega_{* V e} \omega k_{z} \bar{V}_{z} .
$$

Up to a factor of $\gamma$ this is the same as the fluid dispersion relation given by Eq. (46).

\section{Fluid Limit of Kinetic Equations: Nonlocal Analysis}

Nonlocal analytic solutions to the basic eigenmode equation [Eq. (37)] can be obtained in the fluid limit. In particular, the large argument expansion of the plasma dispersion function produces a Weber-type equation with a potential which can be written as a polynomial in $\bar{x}$ where $\bar{x}=x / \rho_{s}$. The parameter regime best suited for this type of analysis is in the limit $|\Omega| \ll K, K b,<1$ and $K s \ll 1$, with $K=\left(1+\eta_{i}\right) / \tau$ and $s=L_{n} / L_{s}$. Expanding the $Z$-functions in the large argument limit then gives

$$
Q(\bar{x})=\left(-b_{s}+\frac{1-\tilde{\Omega}}{\tilde{\Omega}+K}-\frac{\left(\bar{v}_{z} L_{n v}\right) s \bar{x}}{\tilde{\Omega}(\tilde{\Omega}+K)}+\frac{s^{2} \bar{x}^{2}}{\tilde{\Omega}^{2}}\right)
$$

with $\bar{v}_{z}=v_{0 z} / c_{s}, L_{n v}=L_{n} / L_{v}$, and $\tilde{\Omega}=\Omega-\left(\bar{v}_{y} L_{n v}\right) \bar{x}$ where $\bar{v}_{y}=v_{0 y} / c_{s}$. Expanding $Q(\bar{x})$ to $O\left(\bar{x}^{4}\right)$ we get the differential equation

$$
\frac{\partial^{2} \Phi}{\partial \bar{x}^{2}}-\left(A+B \bar{x}+C \bar{x}^{2}+D \bar{x}^{3}+E \bar{x}^{4}\right) \Phi=0
$$

with the coefficients

$$
\begin{aligned}
& A=-\left(-b_{s}+\frac{1}{K+\Omega}-\frac{\Omega}{K+\Omega}\right), \\
& B=-\left(-\frac{\left(\bar{v}_{y} L_{n v}\right)}{(K+\Omega)^{2}}+\frac{\Omega\left(\bar{v}_{y} L_{n v}\right)}{(K+\Omega)^{2}}-\frac{\left(\bar{v}_{y} L_{n v}\right)}{K+\Omega}-\frac{s\left(\bar{v}_{z} L_{n v}\right)}{\Omega(K+\Omega)}\right), \\
& C=-\left(\frac{s^{2}}{\Omega^{2}}+\frac{\left(\bar{v}_{y} L_{n v}\right)^{2}}{(K+\Omega)^{3}}-\frac{\Omega\left(\bar{v}_{y} L_{n v}\right)^{2}}{(K+\Omega)^{3}}+\frac{\left(\bar{v}_{y} L_{n v}\right)^{2}}{(K+\Omega)^{2}}\right.
\end{aligned}
$$




$$
\begin{gathered}
\left.+\frac{s\left(\bar{v}_{y} L_{n v}\right)\left(\bar{v}_{z} L_{n v}\right)}{\Omega(K+\Omega)^{2}}+\frac{s\left(\bar{v}_{y} L_{n v}\right)\left(\bar{v}_{z} L_{n v}\right)}{\Omega^{2}(K+\Omega)}\right) \\
D=-\left(\frac{-2 s^{2}\left(\bar{v}_{y} L_{n v}\right)}{\Omega^{3}}-\frac{\left(\bar{v}_{y} L_{n v}\right)^{3}}{(K+\Omega)^{4}}+\frac{\Omega\left(\bar{v}_{y} L_{n v}\right)^{3}}{(K+\Omega)^{4}}-\frac{\left(\bar{v}_{y} L_{n v}\right)^{3}}{(K+\Omega)^{3}}\right. \\
\left.-\frac{s\left(\bar{v}_{y} L_{n v}\right)^{2}\left(\bar{v}_{z} L_{n v}\right)}{\Omega(K+\Omega)^{3}}-\frac{s\left(\bar{v}_{y} L_{n v}\right)^{2}\left(\bar{v}_{z} L_{n v}\right)}{\Omega^{2}(K+\Omega)^{2}}-\frac{s\left(\bar{v}_{y} L_{n v}\right)^{2}\left(\bar{v}_{z} L_{n v}\right)}{\Omega^{3}(K+\Omega)}\right)
\end{gathered}
$$

and

$$
\begin{aligned}
E= & -\left(\frac{3 s^{2}\left(\bar{v}_{y} L_{n v}\right)^{2}}{\Omega^{4}}+\frac{\left(\bar{v}_{y} L_{n v}\right)^{4}}{(K+\Omega)^{5}}-\frac{\Omega\left(\bar{v}_{y} L_{n v}\right)^{4}}{(K+\Omega)^{5}}+\frac{\left(\bar{v}_{y} L_{n v}\right)^{4}}{(K+\Omega)^{4}}+\frac{s\left(\bar{v}_{y} L_{n v}\right)^{3}\left(\bar{v}_{z} L_{n v}\right)}{\Omega(K+\Omega)^{4}}\right. \\
& \left.+\frac{s\left(\bar{v}_{y} L_{n v}\right)^{3}\left(\bar{v}_{z} L_{n v}\right)}{\Omega^{2}(K+\Omega)^{3}}+\frac{s\left(\bar{v}_{y} L_{n v}\right)^{3}\left(\bar{v}_{z} L_{n v}\right)}{\Omega^{3}(K+\Omega)^{2}}+\frac{s\left(\bar{v}_{y} L_{n v}\right)^{3}\left(\bar{v}_{z} L_{n v}\right)}{\Omega^{4}(K+\Omega)}\right)
\end{aligned}
$$

In the absence of $\hat{y}$-direction velocity shear, the coefficients $D$ and $E$ vanish to lowest order while the other coefficients reduce to

$$
A=-\frac{1}{K} \quad, \quad B=\frac{\left(\bar{v}_{z} L_{n v}\right) s}{\Omega K} \quad, \quad \text { and } \quad C=-\frac{s^{2}}{\Omega^{2}} .
$$

This leads to the eigenvalue condition

$$
\frac{B^{2}}{4 C^{3 / 2}}-\frac{A}{C^{1 / 2}}=1
$$

which in turn gives for the eigenvalue,

$$
\Omega=\frac{i s K}{1-\left(\left(\bar{v}_{z} L_{n v}\right)^{2} / 4 K\right)} .
$$

The result here therefore demonstrates the destabilizing effect of the $\hat{\mathbf{z}}$-direction velocity shear on the mode. ${ }^{2}$ Note that Eq. (5.5) also shows that the effect is independent of the sign of $\left(\bar{v}_{z} L_{n v}\right)$ and that the result is consistent with the earlier assumption, $|\Omega| \ll K$. 

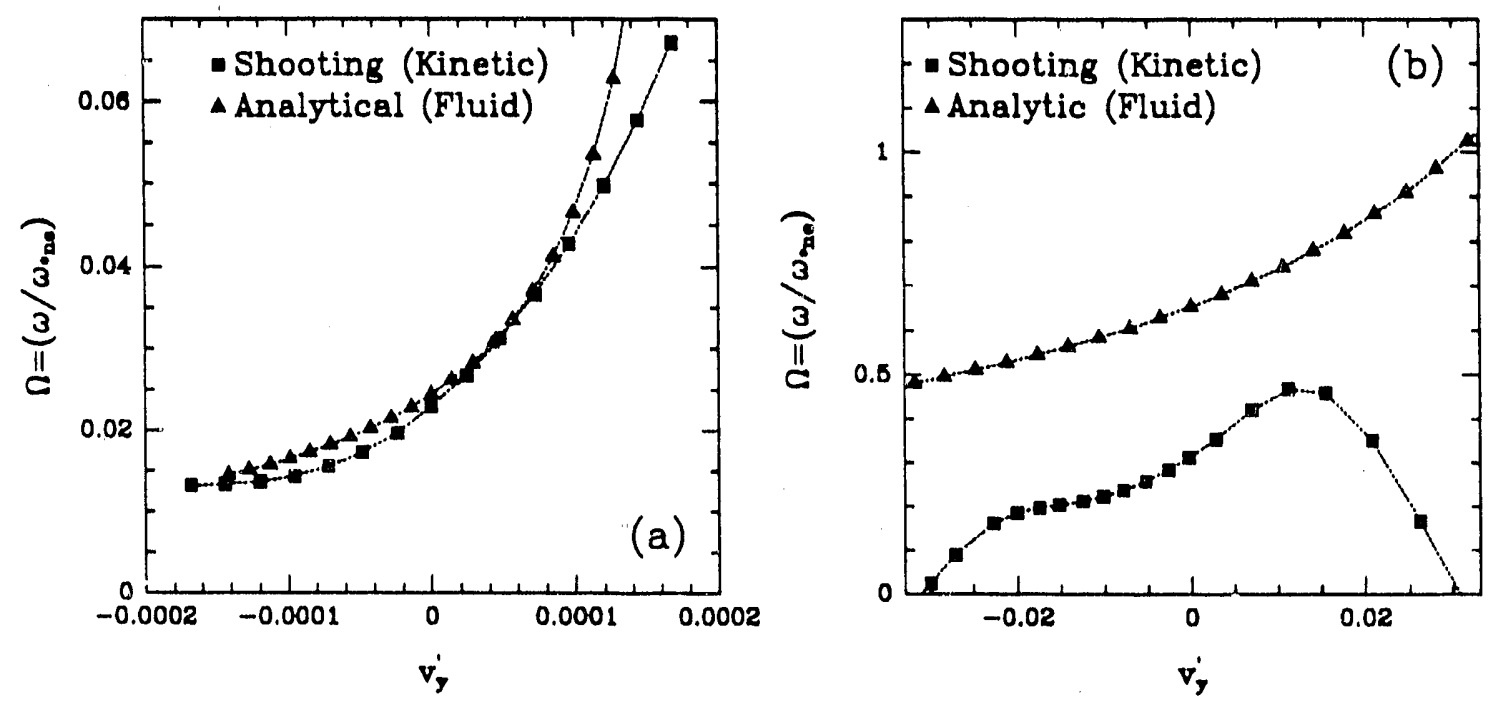

Figure 1: Comparison of the growth rates obtained from analytical estimates and kinetic results (a) for the case $b_{s}=0, \eta_{i}=19, \tau=1, v_{z}^{\prime}=0.3$, and $L_{n} / L_{s}=0.001$ (b) for the case $b_{s}=0.1, \eta_{i}=5 ., L_{n} / L_{s}=0.1, v_{z}^{\prime}=0.2$ 
In order to analyze the effect of the $\hat{\mathbf{y}}$-direction velocity shear on the mode, it is convenient to make the "a priori" assumption that the mode extends to $x=\sqrt{K}$. To justify the expansion, we choose $\left(\bar{v}_{y} L_{n v}\right) \sqrt{K}<\Omega$. For ordering purposes we take $\left(\bar{v}_{y} L_{n v}\right) \sim \Omega / K \sim s$ and also assume that $\left(\bar{v}_{z} L_{n v}\right) \sim O(1)$. The coefficients to lowest order are then given by

$$
\begin{aligned}
& A \simeq-\frac{1}{K}, \\
& B \simeq \frac{\left(\bar{v}_{z} L_{n v}\right) s}{\Omega K}, \\
& C \simeq-\frac{s^{2}}{\Omega^{2}}-\frac{s\left(\bar{v}_{y} L_{n v}\right)\left(\bar{v}_{z} L_{n v}\right)}{\Omega^{2} K}, \\
& D \simeq \frac{2 s^{2}\left(\bar{v}_{y} L_{n v}\right)}{\Omega^{3}}+\frac{s\left(\bar{v}_{y} L_{n v}\right)^{2}\left(\bar{v}_{z} L_{n v}\right)}{\Omega^{3} K},
\end{aligned}
$$

and

$$
E \simeq-\frac{3 s^{2}\left(\bar{v}_{y} L_{n v}\right)^{2}}{\Omega^{4}}-\frac{s\left(\bar{v}_{y} L_{n v}\right)^{3}\left(\bar{v}_{z} L_{n v}\right)^{3}}{\Omega^{4} K} .
$$

The modified eigenvalue condition,

$$
\frac{B^{2}}{4 C^{3 / 2}}-\frac{A}{C^{1 / 2}}=1+\delta
$$

with

$$
\delta=-\frac{11}{16}\left[\frac{D}{C^{5 / 4}}\right]^{2}+\frac{3}{4} \frac{E}{C^{3 / 2}}-\frac{21}{16}\left[\frac{E}{C^{3 / 2}}\right]^{2}
$$

can be obtained following the perturbative analysis outlined in Ref. 3. The solution to Eq. (57) is given by

$$
\Omega=i K s(1+\delta)\left[1-\frac{\left(\bar{v}_{z} L_{n v}\right)^{2}}{4 K}\left(1+\frac{3}{2} \frac{\left(\bar{v}_{y} L_{n v}\right)\left(\bar{v}_{z} L_{n v}\right)}{s K}\right)-\frac{\left(\bar{v}_{y} L_{n v}\right)\left(\bar{v}_{z} L_{n v}\right)}{2 s K}\right]^{-1} .
$$


This result is consistent with the previous "a priori" assumptions, and reduces to the earlier result from Ref. 3 when the $\bar{v}_{z}$ term vanishes.

The new result given in Eq. (59) demonstrates that the inclusion of $v_{y}^{\prime}$ in the problem changes the stability properties through coupling with the toroidal velocity shear. The parameter $\delta$ is symmetric with respect to the relative sign of $v_{y}^{\prime}$ and $v_{z}^{\prime}$ velocity shear and much smaller in magnitude than the rest of the terms in the expression. In Fig. la we provide a comparison of the preceding analytic expression with the full kinetic shooting code result. The factor $\delta$ is ignored here because we found that for the range of $\bar{v}_{y}$ shown in the plot, its inclusion makes no visible change in the results plotted. The good fit of the analytic approximation with the kinetic results is mainly due to the fact that the cases analyzed are far awdy from the marginal stability regime. For more realistic values of $\eta_{i}$ and $L_{n} / L_{s}$, significant divergence from the kinetic results are usually found. Specifically, the analytically calculated growth rate is significantly higher than the growth rate predicted by full kinetic shooting code results. This effect could be due in large part to the absence of the Landau damping term in the fluid approximation. Hence, for a more realistic assessment of growth rates in regimes of interest (e.g., in regions approaching marginal stability) it is necessary to carry out the kinetic analysis.

\section{B. Numerical Results: Kinetic Aralysis}

In the numerical analysis of the kinetic problem we used a WKB shooting code ${ }^{12}$ to solve Eq. (37). The problem is posed in the form

$$
\frac{\partial^{2} \Phi}{\partial x^{2}}+Q(x, \lambda) \Phi=0
$$

After matching the WKB solution at large $x$, the code shoots inward until matching $\Phi$ and $\Phi^{\prime}$ at the origin. The eigenvalue $\lambda$, which in this case corresponds to $\Omega$, is varied until a desired accuracy is reached in the matching condition. 
The sheared flow model used in the present numerical analysis is given by $\mathrm{V}_{0}(\mathrm{x}) \equiv$ $v_{0 z}\left(x / L_{v}\right) \hat{\mathbf{z}}+v_{0 y}\left(x / L_{v}\right) \hat{\mathbf{y}}$. When normalized to $v_{t h_{i}}$ and to the temperature gradient scale length $L_{T}$, the $x$-derivative of the sheared velocity field is given by $v_{y}^{\prime}=\left(v_{0 y} / v_{t h_{i}}\right)\left(L_{T} / L_{v}\right)$ and $v_{z}^{\prime}=\left(v_{0 z} / v_{t h_{i}}\right)\left(L_{T} / L_{v}\right)$. These are designated as the $\hat{\mathbf{y}}$ and $\hat{\mathbf{z}}$ direction flow parameter respectively.

Representative cases for normal, flat, and inverted density profile conditions have been investigated. We chose the flow shear to scale with the temperature gradient, i.e. $L_{T} / L_{v} \simeq 1$ and analyzed cases with $\hat{\mathbf{z}}$-direction flow velocities up to the ion thermal speed and $\hat{\mathbf{y}}$-direction flow velocities of a few percent of the ion thermal speed. Earlier derivations of the equilibrium distribution with sheared flows in toroidal geometry have shown that poloidal flows could not enter into the leading order result. ${ }^{6,13}$ Hence, choosing larger values for $\hat{\mathbf{y}}$-direction flow (i.e.; poloidal flow in a large aspect ratio tokamak) would not be very realistic. The choice to consider $\hat{\mathbf{z}}$-direction flow velocities only up to the ion thermal speed is appropriate since the focus here is on ion temperature gradient modes rather than Kelvin-Helmholtz type instabilities. Moreover, experimental results for poloidal and toroidal rotation from $\mathrm{H}$-mode plasmas ${ }^{5,14-16}$ are consistent with the range of flow parameters considered in the present studies.

\section{Normal Density Gradient Regime}

For a representative normal density gradient case, we chose $\tau=1, b_{s}=0.1$, and $L_{n} / L_{s}=0.1$. In Fig. 2 , the real and imaginary parts of the eigenfrequency $\left(\omega / \omega_{* n e}\right)$ are plotted vs. $\eta_{i}$ for various $v_{z}^{\prime}$ values in the absence of $\hat{\mathbf{y}}$-direction sheared flows $\left(v_{y}^{\prime}=0\right)$. The cases considered indicate that the mode is destabilized for even small values of the flow parameter $v_{z}^{\prime}$. Although the change in the $\eta_{i}$-threshold is not significant, once excited, the ion temperature gradient instability is clearly stronger in the presence of $\hat{\mathbf{z}}$-direction sheared flows. 

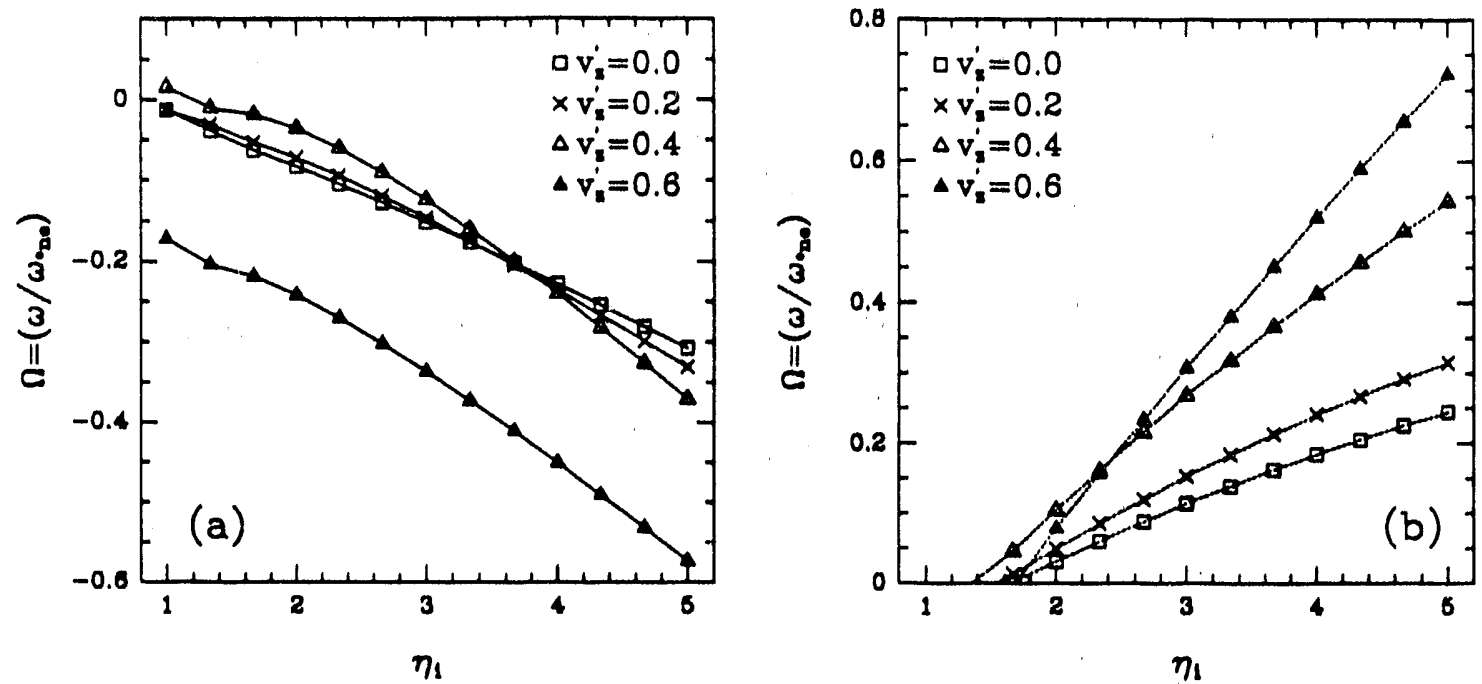

Figure 2: Eigenfrequency vs. $\eta_{i}$ for the positive density gradient case with $\tau=1, b_{s}=0.1$, $v_{y}^{\prime}=0$, and $L_{n} / L_{s}=0.1 ;$ (a) real part (b) imaginary part.
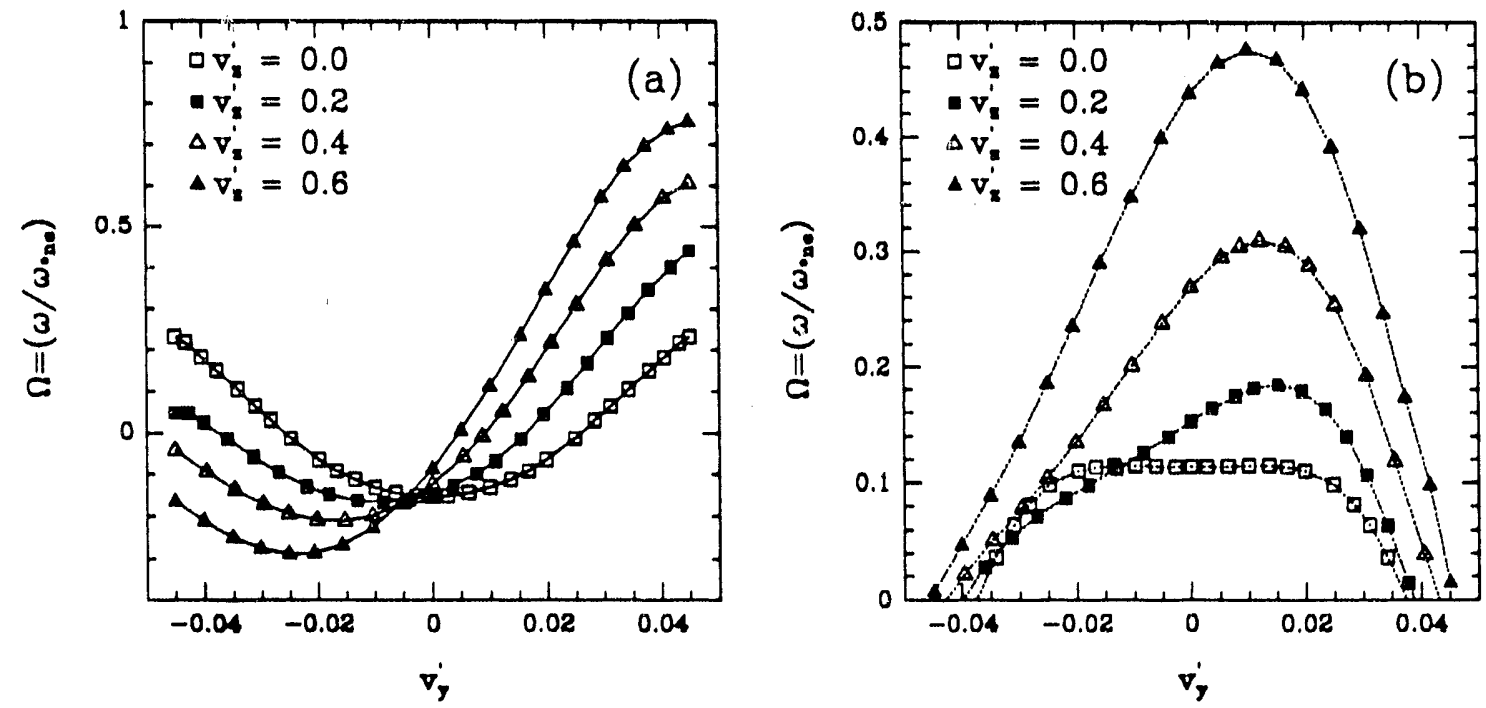

Figure 3: Eigenfrequency vs. $v_{y}^{\prime}$ for the positive density gradient case with $\eta_{i}=3, \tau=1$., $b_{s}=0.1$, and $L_{n} / L_{s}=0.1$; (a) real part (b) imaginary part. 

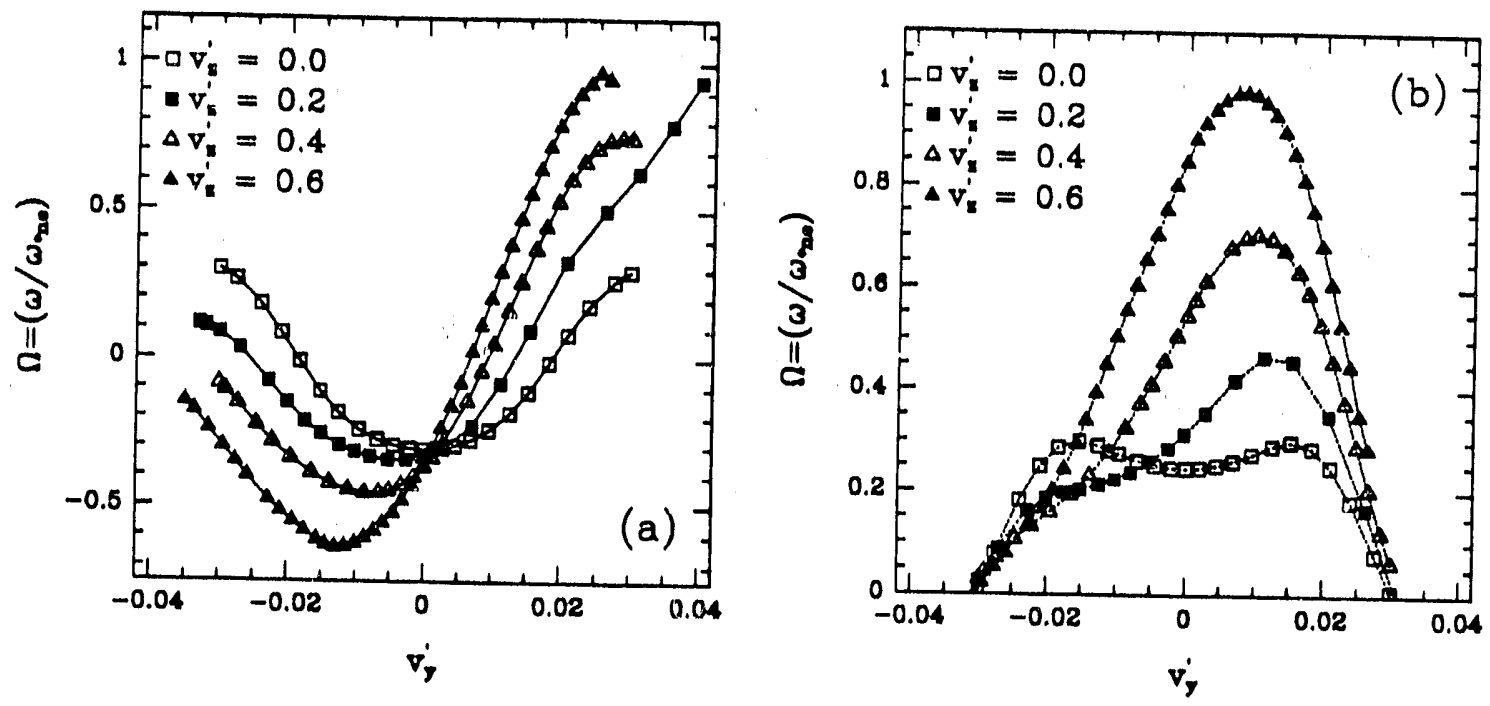

Figure 4: Eigenfrequency vs. $v_{y}^{\prime}$ for the positive density gradient case with $\eta_{i}=5, \tau=1$, $b_{s}=0.1$, and $L_{n} / L_{s}=0.1$; (a) real part (b) imaginary part.

Interesting features are produced by the introduction of $\hat{\mathbf{y}}$-direction flows in the problem. In Fig. 3 the eigenfrequency is plotted vs. the the flow parameter $v_{y}^{\prime}$ for various values of the flow narameter $v_{z}^{\prime}$ with $\eta_{i}=3$. Figure 4 examines the same trends for $\eta_{i}=5$. Concentrating on the $v_{z}^{\prime}=0$ case in Fig. $4 \mathrm{~b}$, we observe a slight destabilizing trend around $v_{y}^{\prime}=0$, which is less pronounced in the $\eta_{i}=3$ case (Fig. $3 \mathrm{~b}$ ). The expansion of the kernel [Eq. (37)] to $O\left(x^{3}\right)$ gives rise to perturbative corrections to the eigenvalue [Eq. (58)]. These analytical estimates qualitatively agree with our findings for $\eta_{i} \gg 1$. However, for $\eta_{i}=3$ [i.e., closer to the marginal stability region] this trend almost completely vanishes. A possible explanation of this might come from the fact that kinetic effects dominate near marginal stability. Specifically, the ion Landau damping absent in fluid estimates becomes important here. For cases with $v_{z}^{\prime} \neq 0$ we observe an asymmetry of the growth rate with respect to the sign of the $\hat{y}$-direction flow parameter $v_{y}^{\prime}$. Examination of the kernel used in the shooting code [Eq. (37)] indicates that a sign change for 


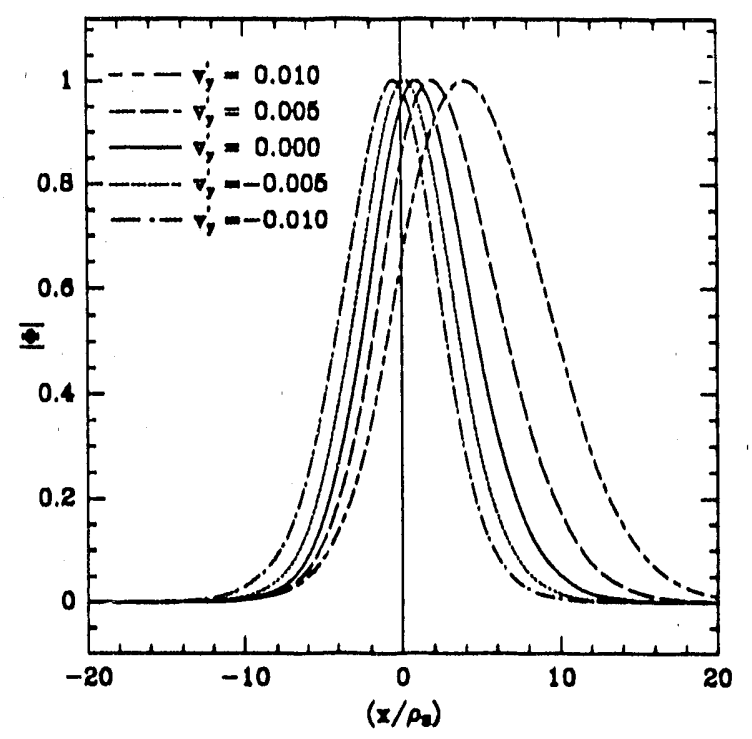

Figure 5: Plot of the eigenfunction for $\eta_{i}=5, L_{n} / L_{s}=0.1, b_{s}=0.1, v_{z}^{\prime}=0.2$ and $\tau=1$. $v_{z}^{\prime}$ correspondingly flips the symmetry of the mode in the $\hat{\mathbf{x}}$-direction. In addition, the mode is sensitive to the relative sign of $v_{z}^{\prime}$ and $v_{y}^{\prime}$, the $\hat{\mathbf{z}}$ and $\hat{\mathbf{y}}$ direction flow parameters respectively. This can be stated as follows:

$$
\left.\Omega\left(v_{z}^{\prime}, v_{y}^{\prime}\right)=\Omega\left(-v_{z}^{\prime},-v_{y}^{\prime}\right) \quad \text { and } \quad \Phi\left(x, v_{z}^{\prime}, v_{y}^{\prime}\right)=\Phi\left(-x,-v_{z}^{\prime},-v_{y}^{\prime}\right)\right)
$$

where $\Omega$ and $\Phi$ are the eigenfrequency and eigenfunction respectively. For every $v_{z}^{\prime}$ there is a corresponding $v_{y}^{\prime}$ that can maximize the growth rate. It is found that this occurs for $v_{z}^{\prime} v_{y}^{\prime}>0$.

Regarding the question of how sheared flows affect the threshold for ITG modes, we have examined in particular the effect of parallel ( $\hat{\mathbf{z}}$-direction) sheared flows on the stabilizing influence of perpendicular ( $\hat{\mathbf{y}}$-direction) sheared flows. A comparison of Figs. $3 \mathrm{~b}$ and $4 \mathrm{~b}$ shows that in the $\eta_{i}=3$ case the threshold $v_{y}^{\prime}$ values for complete stabilization are dependent on $v_{z}^{\prime}$. However, for $\eta_{i}=5$, the trend is less pronounced and the threshold values are not significantly different for various $v_{z}^{\prime}$. This again might be attributable to the enhanced role of kinetic effects as the marginal stability region (i.e., $\eta_{i}=3$ ) is approached. 

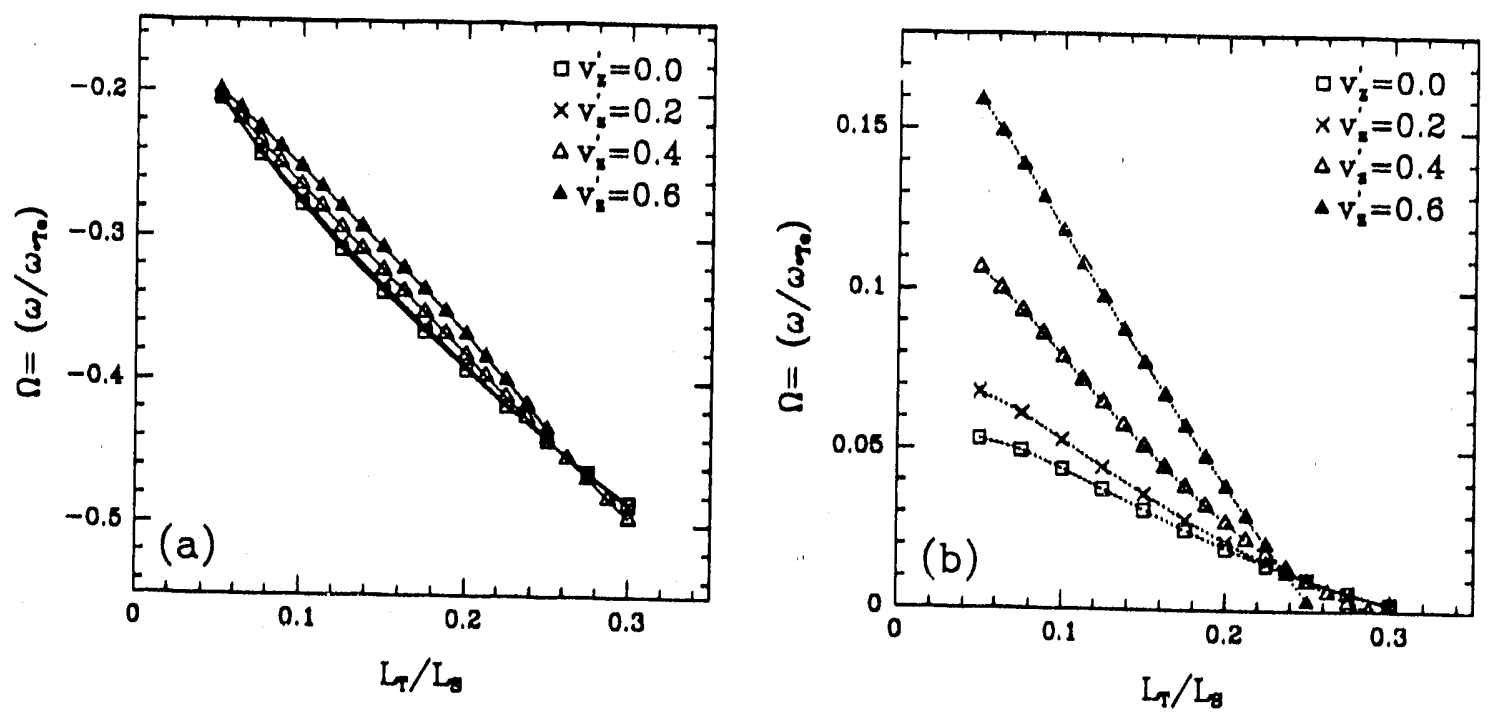

Figure 6: Eigenfrequency for the flat density gradient case with $b_{0}=0.1, \tau=1$, and $v_{y}^{\prime}=0 ;(\mathrm{a})$ real part (b) imaginary part.

The influence of sheared flow effects on the mode structure of ITG instabilities is illustrated in Fig. 5. For a representative case with sheared flow in the $\mathbf{z}$-direction $\left(v_{z}^{\prime}=\right.$ $0.2)$, it is seen that the eigenfunction tends to be more localized in the $x>0$ region as the poloidal shear $\left(v_{y}^{\prime}\right)$ goes from negative to zero to positive values. Since $v_{y}^{\prime} / v_{z}^{\prime}>0$ corresponds to the most unstable situations (see Fig. 4) and since $B_{y} / B_{z}>0$ for $x>0$ for sheared slab models, these results suggest that ITG modes tend to be stronger when the sheared flows are more aligned with the equilibrium magnetic field in the region where the mode is localized. However, for sufficiently large values of $v_{y}^{\prime}$, the stabilizing influence is present regardless of the sign of $v_{z}^{\prime} v_{y}^{\prime}$.

\section{Flat Density Gradient Regime}

It is well known that flat density profiles are a common feature in a large portion of most $\mathrm{H}$-mode plasmas. Here we investigate a representative flat density gradient case 

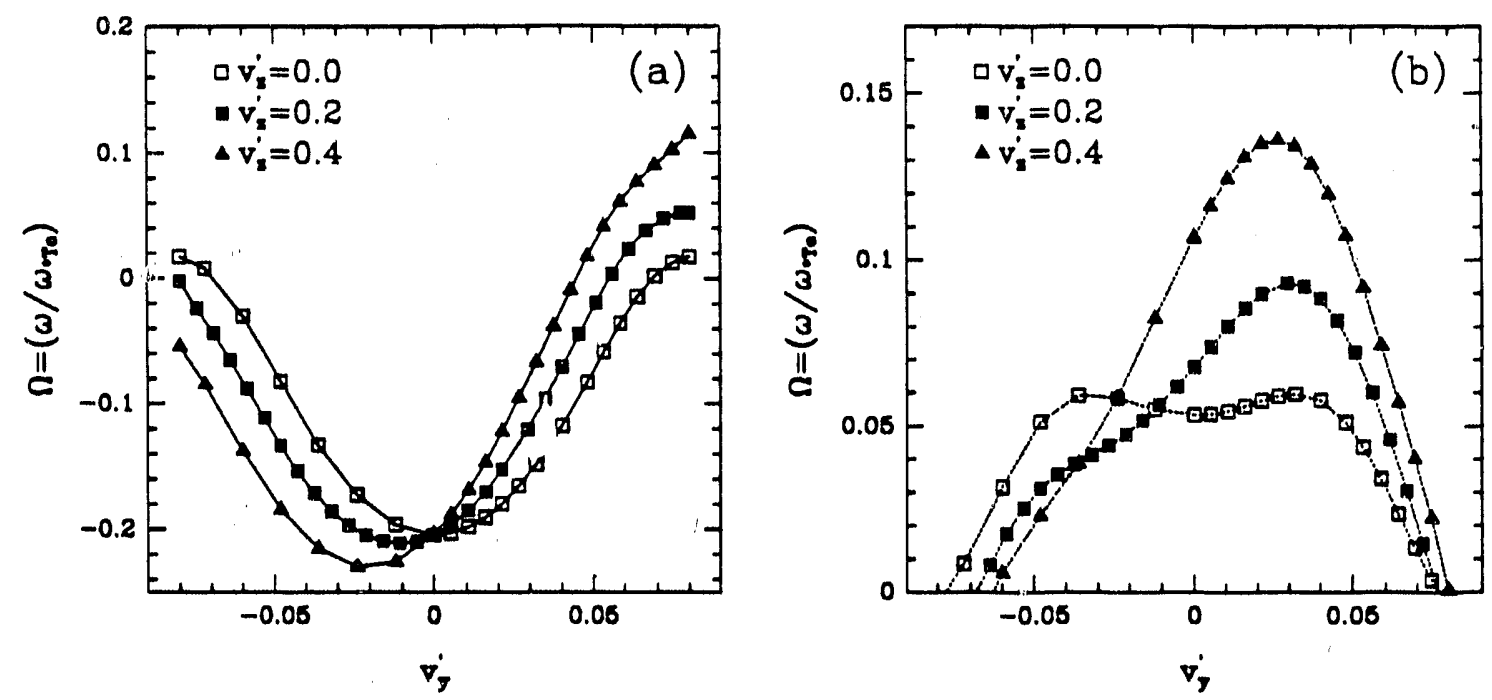

Figure 7: Eigenfrequency vs. $v_{y}^{\prime}$ for the flat density gradient case with $L_{T} / L_{s}=0.05$, $b_{\mathbf{s}}=0.1$, and $\tau=1 ;(\mathrm{a})$ real part (b) imaginary part.

with the parameters $\tau=1$, and $b_{s}=0.1$. Unlike the normal and inverted density gradient cases, the eigenfrequency here is normalized against $\omega_{*} T_{e}$. In Fig. 6 we plot the eigenfrequency vs. $L_{T} / L_{s}$ for various $\hat{z}$-direction flow parameters $v_{z}^{\prime}$ in the absence of $\hat{y}$-direction sheared flows [i.e.; $v_{y}^{\prime}=0$ ]. Fig. $6 \mathrm{~b}$ indicates a very modest favorable influence of $v_{z}^{\prime}$ in that the threshold for the temperature gradient $\left(L_{T} / L_{s}\right)$ slightly shifts to the left, thereby allowing steeper temperature profiles. However, similar to the normal and inverted density gradient cases, the instability, once excited, is stronger in the presence of sheared flows in the $\hat{\mathbf{z}}$-direction. Including $\hat{\mathbf{y}}$-direction sheared flows (Fig. $\mathbf{\tau}$ ) does not significantly change the picture from those found in the normal and inverted density gradient cases. The slight destabilization for small values of $v_{y}^{\prime}$ in the absence of $\hat{\mathbf{z}}$-direction flow is visible here, and the growth rate curve for $v_{z}^{\prime}=0.2$ also exhibits this trend in the vicinity around $v_{y}^{\prime}=0$. In these flat density profile cases the threshold $v_{y}^{\prime}$ values again exhibit an asymmetry with respect to the sign of $v_{y}^{\prime}$. On the positive side, i.e., for $v_{y}^{\prime}>0$, 

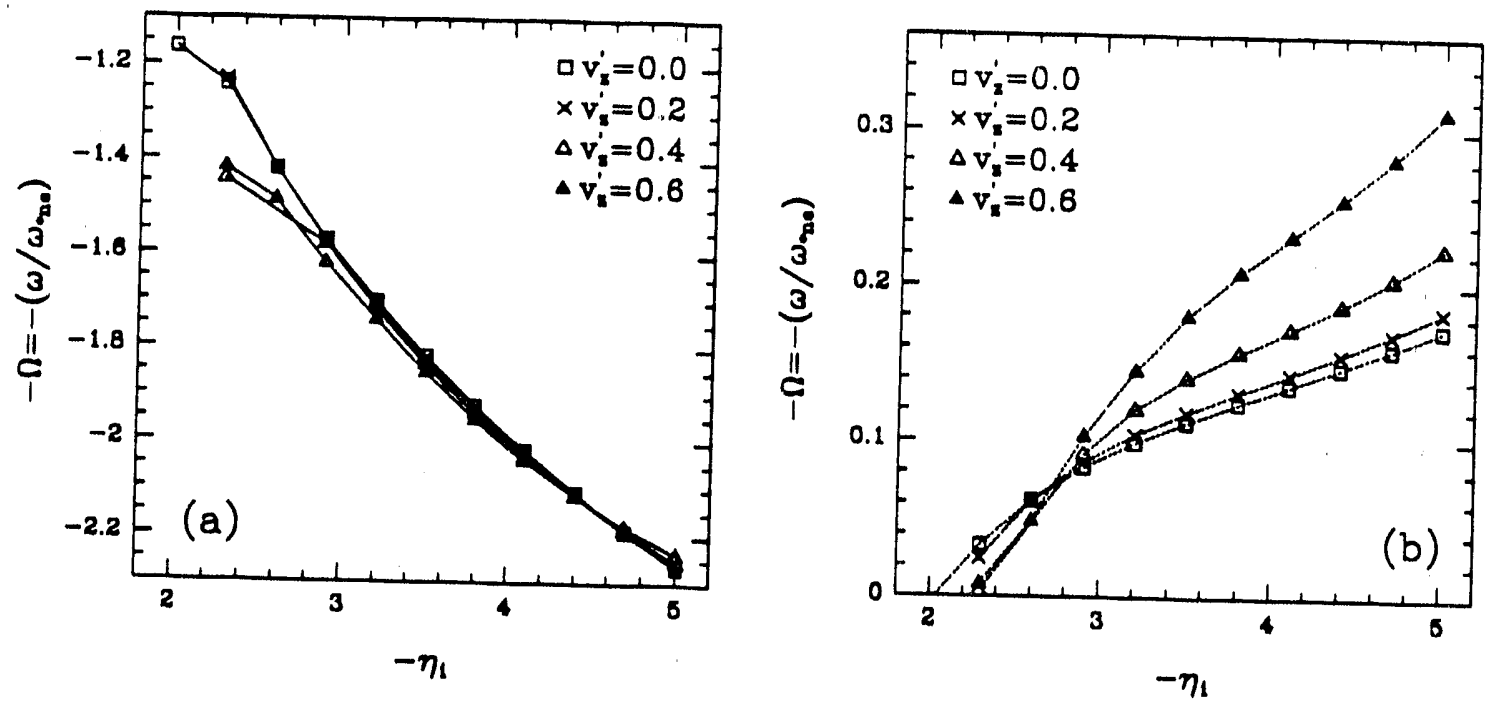

Figure 8: Eigenfrequency vs. $\eta_{i}$ for the inverted density gradient case with $L_{n} / L_{s}=-1$, $b_{s}=0.1$, and $v_{y}^{\prime}=0 ;(\mathrm{a})$ real part (b) imaginary part.

larger values of $v_{y}^{\prime}$ are required to stabilize modes with larger $v_{z}^{\prime}$ values. For the opposite situation smaller $v_{y}^{\prime}$ values are sufficient to stabilize modes with larger $v_{z}^{\prime}$. Again, for a given $v_{z}^{\prime}$, there will be a value of $v_{y}^{\prime}$ which will maximize the growth rate.

\section{Inverted Density Gradient Regime}

In addition to flat density profiles, $\mathrm{H}$-mode experimental results from tokamaks such as DIII-D ${ }^{15}$ and JET $^{17}$ have also exhibited cases where the density profiles can be outwardly peaked. In examining such negative $\eta_{i}$ scenarios, we will consider $L_{n} / L_{s}=-1$. The rest of the parameters are the same as in the normal density gradient case with $b_{s}=0.1$, and $\tau=1$. In Fig. 8 the eigenfrequency is plotted vs. $\eta_{i}$ for various values of the $\hat{\mathbf{z}}$-direction flow parameter $v_{z}^{\prime}$ with $v_{y}^{\prime}=0$. We observe a slight favorable shift in the $\eta_{i}$ threshold. However, as in the other cases, once the instability is excited, it exhibits larger growth rates. Figure 9 indicates that $\hat{y} \cdot$ direction sheared flow uniformly stabilizes the mode even 

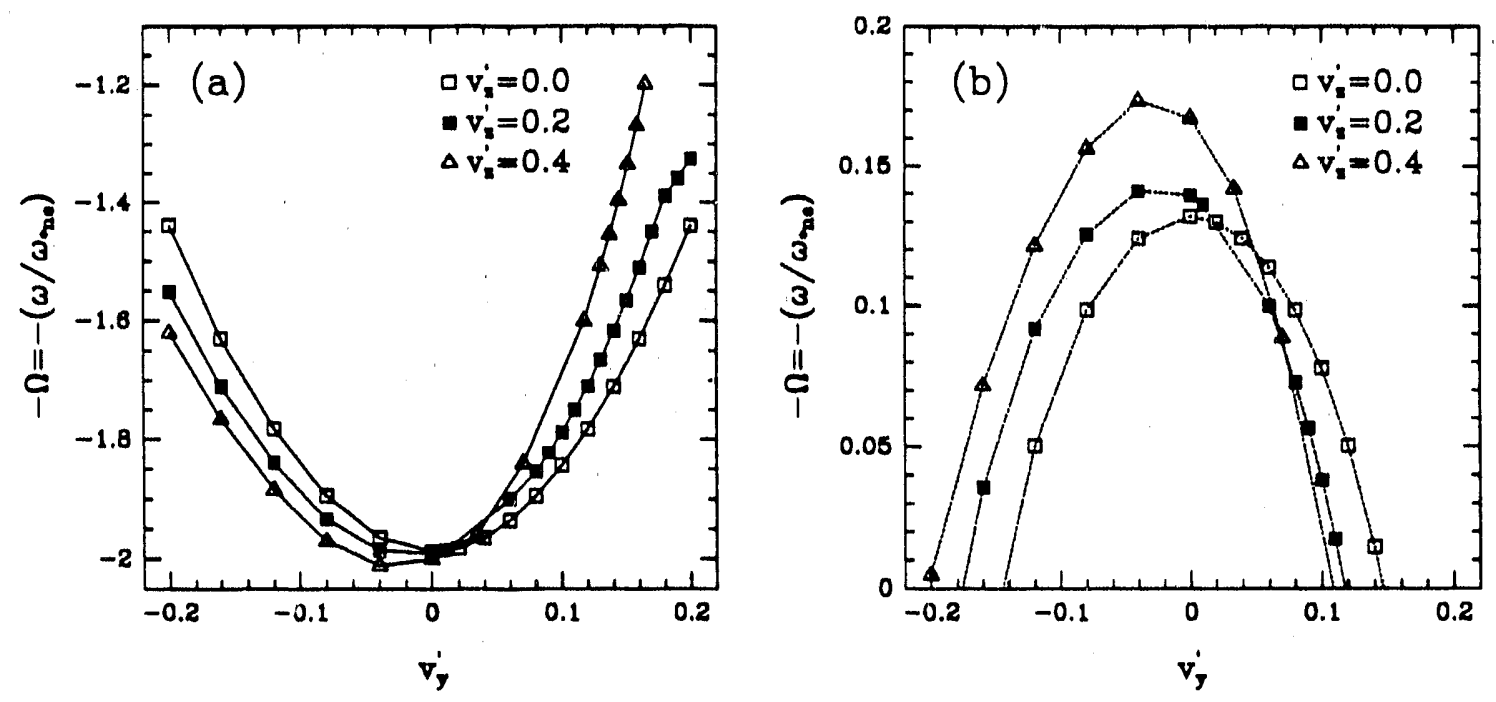

Figure 9: Eigenfrequency vs. $v_{y}^{\prime}$ for the inverted density gradient case with $L_{n} / L_{s}=-1$, $b_{s}=0.1$, and $\eta_{i}=-4$; (a) real part (b) imaginary part.

in the absence of the $\hat{\mathbf{z}}$-direction sheared flow. Similar to the flat density gradient case, the threshold values for $v_{y}^{\prime}$ exhibit an asymetry with respect to the sign of the flow parameter. Here, on the positive side, smaller values of $v_{y}^{\prime}$ are sufficient to stabilize modes with larger values of $v_{z}^{\prime}$. For the opposite case (i.e., $v_{y}^{\prime}<0$ ) the opposite trend is observed. 


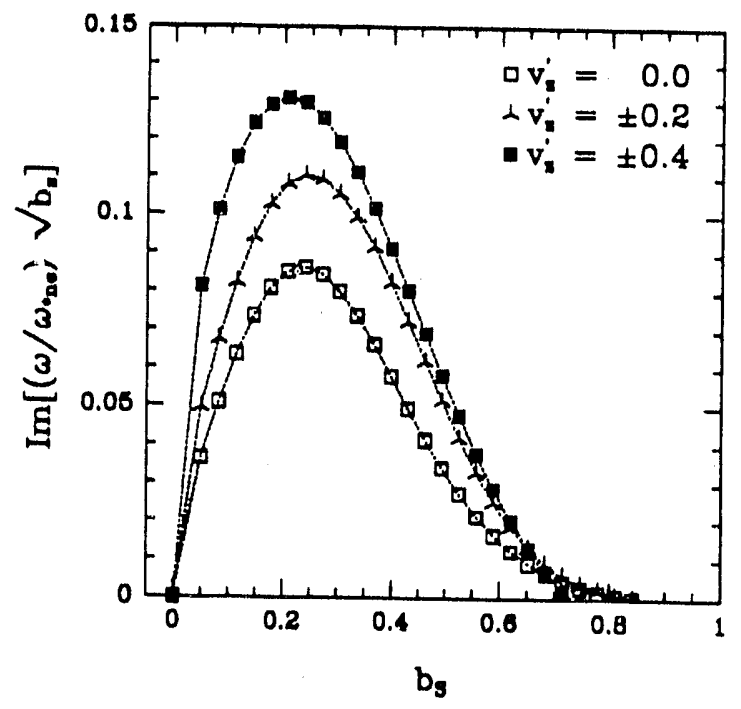

Figure 10: $k_{y}$-spectrum (growth rate vs. $b_{s}$ ) for $v_{y}^{\prime}=0, \eta_{i}=4, \tau=1$ and $L_{n} / L_{s}=0.1$.

\section{Summary and Discussion}

In this paper we have presented a systematic kinetic analysis of the combined influence of sheared equilibrium flows (in the $\hat{\mathbf{y}}$ and $\hat{\mathbf{z}}$ direction) on ion temperature gradient instabilities for a sheared slab geometry. The theoretical foundation for these studies was provided by a rigorous derivation of the linearized gyrokinetic equations in the presence of such flows. As in earlier fluid limit studies, ${ }^{2,3}$ we find that parallel ( $\hat{\mathbf{z}}$-direction) velocity shear tends to be destabilizing and perpendicular ( $\hat{y}$-direction) sheared flows can completely stabilize the ITG modes for sufficiently large values of the flow parameter $v_{y}^{\prime}$. However, the most realistic scenario is when both types of sheared flow are simultaneously present. We have accordingly focused our analysis on this issue with results indicating a new trend; i.e., in the presence of $\hat{\mathbf{z}}$-direction sheared flow, $\hat{\mathbf{y}}$-direction sheared flow can be either stabilizing or destabilizing in the vicinity of $v_{y}^{\prime}=0$ depending on the relative sight of these flows. In particular, $\hat{\mathbf{y}}$-direction sheared flows can be stabilizing when $v_{z}^{\prime} v_{y}^{\prime}<0$ 

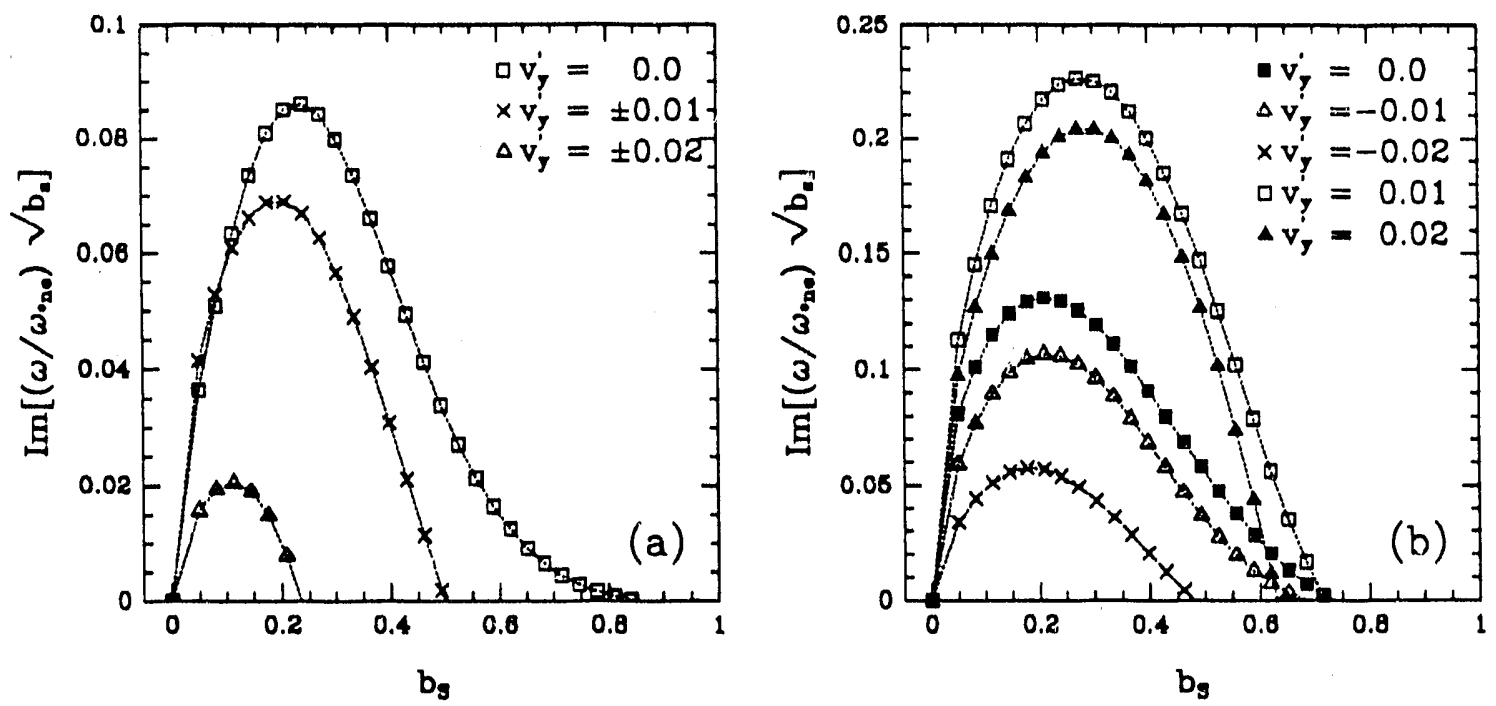

Figure 11: $k_{y}$-spectrum (growth rate vs. $b_{s}$ ) for $\eta_{i}=4, \tau=1, L_{n} / L_{s}=0.1$, and (a) $v_{z}^{\prime}=0$ (b) $v_{z}^{\prime}=0.4$.

and destabilizing when $v_{z}^{\prime} v_{y}^{\prime}>0$. However, for sufficiently large values of $v_{y}^{\prime}$ the mode is completely stabilized regardless of the $\mathrm{s}^{\prime} \mathrm{gn}$ of $v_{z}^{\prime} v_{y}^{\prime}$. The actual threshold $v_{y}^{\prime}$ value required for stabilization is weakly sensitive to the magnitude and sign of this quantity.

With regard to the relationship of these slab results to tokamak geometry, some additional comments are appropriate. The identification of the $\hat{z}$-direction with the toroidal direction and the $\hat{y}$-direction with the poloidal direction is rigorously correct up to order $\epsilon / q$, the angle between the parallel and toroidal directions in a tokamak. If we choose to associate the parallel direction in a tokamak with the $\hat{\mathbf{z}}$-direction in a slab, then the $\hat{\mathbf{y}}$-direction component of the toroidal flow is $\epsilon / q$ times smaller than the $\hat{\mathbf{z}}$-direction part. If the ordering is such that $\epsilon / q$ is less than $\rho_{i} / L$ the identification of the $\hat{\mathbf{z}}$ and $\hat{\mathbf{y}}$ directions with the toroidal and poloidal directions follows. For cases where $\epsilon / q \simeq \rho_{i} / L$, it is possible for a pure toroidal sheared flow to have a large enough component in the $\hat{y}$-direction to completely stabilize the ITG mode. However, it should be remembered that in this range 
of $\epsilon / q$, it would be necessary to include toroidal field line curvature and trapped particle effects to properly assess stability properties.

Another point of emphasis in the present paper is the importance of a proper kinetic treatment of this problem. Although fluid estimates are useful in providing insight into the general trends associated with sheared flow effects on ITG modes, important quantitative issues, such as establishing stability thresholds and the determination of unstable spectral ranges, clearly require a kinetic analysis. An aspect of this point is illustrated in Fig. 1. In Fig. 1a it is shown that for parameters chosen to satisfy fluid approximations, the kinetic and fluid results are consistent. However, for the more realistic parameters of Fig. 1b, there is an obviously large deviation. Unstable spectral range results, such as those shown on Figs. 10 and 11, can only be reliably obtained through kinetic analysis.

Unstable spectral ranges for ITG modes in the presence of sheared flows are displayed for representative positive gradient $\left(\eta_{i}>0\right)$ cases in Figs. 10 and 11 . This type of information can be useful in the formulation of nonlinear theories in that, as shown in Fig. 11, sheared poloidal flows (for $v_{z}^{\prime}=0$ or $v_{z}^{\prime} v_{y}^{\prime}<0$ ) can act to cut off the unstable range at short wavelengths. For unfavorable situations (i.e., $v_{\nu}^{\prime}=0$ or $v_{z}^{\prime} v_{y}^{\prime}>0$ ), Figs. 10 and $11 \mathrm{~b}$ indicate that although the instabilities are stronger, the spectral range is not significantly modified.

\section{Acknowledgements}

The authors are grateful to T. S. Hahm and S. C. Cowley for many useful discussions and also thank R. B. White for the use of his WKB shooting code.

This work is supported by U.S. Department of Energy Contract No. DE-AC02-76CH03073. 


\section{References}

${ }^{1}$ T. S. Hahm and W. M. Tang, Physics of Fluids 31, 1185 (1988).

${ }^{2}$ N. Mattor and P. H. Diamond, Physics of Fluids 31, 1180 (1988).

${ }^{3}$ X. H. Wang and P. H. Diamond, Stability of Ion Temperature Gradient Driven Modes in the Presence of Sheared Poloidal Flows, submitted to Phyiscs of Fluids B., 1991.

${ }^{4}$ G. M. Staebler and R. R. Dominguez, Electric field effects on ion temperature gradient modes in a sheared slab, submitted to Physics of Fluids B, 1991.

${ }^{5}$ R. J. Groebner, K. H. Burrell, and R. P. Seraydarian, Physical Review Letters 64, 3015 (1990).

${ }^{6}$ P. J. Catto, I. B. Bernstein, and M. Tessarotto, Physics of Fluids 30, 2784 (1987).

${ }^{7}$ P. J. Catto, Plasma Physics 20, 719 (1978).

${ }^{8}$ P. J. Catto, W. M. Tang, and D. E. Baldwin, Plasma Physics 23, 639 (1981).

${ }^{9}$ W. W. Lee, Physics of Fluids 26, 556 (1983).

${ }^{10} \mathrm{~L}$. Chen, Waves and Instabilities in Plasmas, volume 12 of World Scientific Lecture Notes in Physics, World Scientific, Teaneck, New Jersey, 1987.

${ }^{11}$ J. Q. Dong, P. N. Guzdar, and Y. C. Lee, Physics of Fluids 30, 2694 (1987).

${ }^{12}$ R. B. White, Journal of Computational Physics 31(3) (1979).

${ }^{13} \mathrm{~S}$. C. Cowley, private communication.

${ }^{14}$ K. Ida, S. Hidekuma, Y. Miura, T. Fujita, M. Mori, K. Hoshino, N. Suzuki, T. Yamauchi, and the JFT-2M Group, Physical Review Letters 65, 1364 (1990). 
${ }^{15}$ K. H. Burrell, T. N. Carlstorm, E. J. Doyle, P. Gohil, R. J. Groebner, T. Lehecka, N. C. Luhmann, Jr., H. Matsumoto, T. H. Osborne, W. A. Peebles, and R. Philipona, Physics of Fluids B 2, 1405 (1990).

${ }^{16}$ R. J. Taylor, M. L. Brown, B. D. Fried, H. Grote, J. R. Liberati, G. J. Morales, P. Pribyl, D. Darrow, and M. Ono, Physical Review Letters 63, 2365 (1989).

${ }^{17}$ C. Gowers, D. Barlett, A. Boileau, S. Corti, A. Edwards, N. Gottardi, K. Hirsch, M. Keilhacker, E. Lazzaro, P. Morgan, P. Nielsen, J. O'Rourke, H. Salzmann, P. Smeulders, A. Tanga, and M. von Hellermann, in Proceedings of the 15th European Conference on Controlled Fusion and Plasma Heating (European Physical Society, Budapest, 1988), Vol 12B, Part 1, p.2239. 


\section{List of Figures}

1 Comparison of the growth rates obtained from analytical estimates and kinetic results (a) for the case $b_{s}=0, \eta_{i}=19, \tau=1, v_{z}^{\prime}=0.3$, and $L_{n} / L_{s}=0.001$ (b) for the case $b_{s}=0.1, \eta_{i}=5 ., L_{n} / L_{s}=0.1, v_{z}^{\prime}=0.2 \ldots 16$

2 Eigenfrequency vs. $\eta_{i}$ for the positive density gradient case with $\tau=1$, $b_{s}=0.1, v_{y}^{\prime}=0$, and $L_{n} / L_{s}=0.1 ;$ (a) real part (b) imaginary part. . . . 19

3 Eigenfrequency vs. $v_{y}^{\prime}$ for the positive density gradient case with $\eta_{i}=3$, $\tau=1 ., b_{s}=0.1$, and $L_{n} / L_{s}=0.1 ;$ (a) real part (b) imaginary part. . . . . 19

4 Eigenfrequency vs. $v_{y}^{\prime}$ for the positive density gradient case with $\eta_{i}=5$, $\tau=1, b_{s}=0.1$, and $L_{n} / L_{s}=0.1 ;$ (a) real part (b) imaginary part. . . . .

5 Plot of the eigenfunction for $\eta_{i}=5, L_{n} / L_{s}=0.1, b_{s}=0.1, v_{z}^{\prime}=0.2$ and $\tau=1$

6 Eigenfrequency for the flat density gradient case with $b_{s}=0.1, \tau=1$, and $v_{y}^{\prime}=0 ;(\mathrm{a})$ real part (b) imaginary part.

7 Eigenfrequency vs. $v_{y}^{\prime}$ for the flat density gradient case with $L_{T} / L_{s}=0.05$, $b_{s}=0.1$, and $\tau=1 ;(\mathrm{a})$ real part (b) imaginary part.

8 Eigenfrequency vs. $\eta_{i}$ for the inverted density gradient case with $L_{n} / L_{s}=$ $-1, b_{s}=0.1$, and $v_{y}^{\prime}=0 ;(\mathrm{a})$ real part (b) imaginary part.

9 Eigenfrequency vs. $v_{y}^{\prime}$ for the inverted density gradient case with $L_{n} / L_{s}=$ $-1, b_{s}=0.1$, and $\eta_{i}=-4 ;$ (a) real part (b) imaginary part. . . . . . . . 24

$10 k_{y}$-spectrum (growth rate vs. $b_{s}$ ) for $v_{y}^{\prime}=0, \eta_{i}=4, \tau=1$ and $L_{n} / L_{s}=0.1 .26$

$1.1 k_{y}$-spectrum (growth rate vs. $b_{s}$ ) for $\eta_{i}=4, \tau=1, L_{n} / L_{s}=0.1$, and (a)

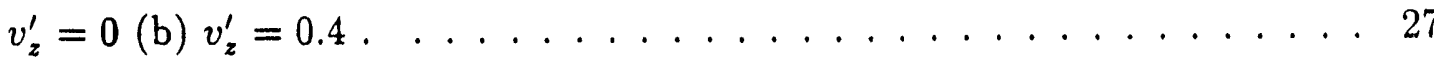


Dr. F. Paoloni, Univ. of Wollongong, AUSTRALIA

Prof. M.H. Brennen, Univ. of Sydney, AUSTRALIA

Plasma Research Lob., Australian Nat. Univ., AUSTRALIA

Prof. I.A. Jones, Flinders Univ, AUSTRALIA

Prof. F. Cap, Inst. for Theoretical Physics, AUSTRIA

Prof. M. Heindler, Institut for Theoretische Physik, AUSTRIA

Prof. M. Goossens, Astronomisch Instituut, BELGIUM

Ecole Royale Militaire, Lab. de Phy. Plasmas, BELGIUM

Commission-European, DG. XII-Fusion Prog., BELGIUM

Prot. R. Bouciquie, Rijksuniverateit Gent, BELGIUM

Dr. P.H. Sakanaka, Instituto Fisica, BRAZIL

Instituto De Pesquiess Eepeciais-INPE, BPAZIL

Documents Office, Acomic Eneroy of Cenada Lid., CANADA

Dr. M.P. Bectynnki, MPB Technologios, Inc., CANADA

Dr. H.M. Skarsgard, Univ. of Sackatchewan, CANADA

Prof. J. Teichmenn, Univ. of Montreal, CANADA

Prof. S.R. Sroenivasan, Univ. of Calgary, CANADA

Prof. T.W. Johnsion, INRS-Energio, CANADA

Dr. R. Bolton, Centre canacien de husion magnótique, CANADA

Dr. C.R. James, Univ. of Nborta, CANADA

Dr. P. Lukac, Komenekeho Universzita, CZECHOSLOVAKIA

The Librarian, Culham Laboratory, ENGLAND

Library, R61, Rutherford Appleton Leboratory, ENGLAND

Mre. S.A. Hutchinson, JET Library, ENGLAND

P. Mthonen, Univ. of Helsink, FINLAND

C. Moutiot, Lab. de Physique des Milieux lonieds, FRANCE

J. Radot, CEN/CADARACHE - Bnt 506, FRANCE

Ms. C. Rinni, Univ, of loamina, GREECE

Dr. T. Mul, Acadermy Bibliographic Ser., HONG KONG

Preprint Librery, Hunowion Academy of Sci., HUNGARY

Dr. B. Das Gupan Satrat Inct of Nudear Physics, INDIA

Dr. P. Kew, Inst for Pleama Posearch, INDIA

Or. P. Rosenau, lereel lnat of Tectnology, ISRAEL

Librarian, International Conter for Thec Physics, ITALY

Miss C. Do Palo, Associazione EURATOM-ENEA, ITALY

Dr. G. Groses, Istituto di Fisica del Plasma, ITALY

Dr. H. Yamato, Toshiba Res \& Devel Center, JAPAN

Prof. I. Kawakami, Atomic Energy Ros. Inst., JAPAN

Prof. K. Nishikewa, Hiroshima Univ., JAPAN
Director, Japan Atomic Energy Research inst., JAPAN

Prof. S. Itoh, Kyustu Univ., JAPAN

Data and Planning Conter, Nagoya Univ., JAPAN

Prof. S. Tanaka, Kyoto Univ., JAPAN

Library, Kyoto Univ., JAPAN

Prof. N. Inoue, Univ. of Tokyo, JAPAN

S. Mori, Technical Advisor, JAEAI, JAPAN

O. Mitara, Kumamoto Inst. of Tectmology, JAPAN

H. Jeong, Korea Autvanced Energy Recearch Inst., KOREA

Prof. D.I. Choi, The Korea Adv. Inst. of Sa. \& Tech., KOREA

Prot. B.S. Liley, Univ. of Waiketo, NEW ZEALAND

Inst. of Plasma Physica, PEOPLE'S REPUBLIC OF CHINA

Librenien, Inst. of Physics. PEOPLE'S REPUBLC OF CHINA

Librery, Tringhun Univ., PEOPLE'S REPUBLIC OF CHINA

2. Li, S.W. Inet Physics, PEOPLE'S REPUBLIC OF CHINA

Prof. J.A.C. Cebral, Institub Superior Tecnico, PORTUGAL

Dr. O. Potrus, AL I CUzA Univ., POMANIA

Dr. J. do Villiers, Fusion Suctes, AEC, S. AFAICA

Prot. M.A. Heibory, Univ. of Natal, S. AFRICA

C.I.E.M.A.T, Fuaion Diviaion Librery, SPAIN

Dr. L Sionflo, Univ. of UMEA, SWEDEN

Librery, Royed Inst. of Technoboy, SWEDEN

Prof. H. Witholmson, Chaimers Univ. of Tech., SWEDEN

Contre Phys. Dos Plasmas, Ecole Polytech, SWIZERLAND

Bibliotheak, Inst. Voor Plasma-Fysica, THE NETHERLANDS

N. Durgut, Vice Chairmen, Midd East Tech. Univ., TURKEY

Dr. D.D. Ayubry, Siberian Brench of Academy of Sci., USSA

Dr. G.A. Eliscov, Kurchatov Inat, USSR

Librarian, The Ukr.SSR Acadeny of Scionces, USSP

Dr. LM. Kovrizhnykh, Inst. of General Physics, USSR

Kemforcchungsanlego GmbH, Zentrabibliothek, W. GERMANY

Bibliothok, Inat. For Plasmatorschung, W. GERMANY

Prof. K. Schinder, Ruhr-Univernitht Bochum, W. GERMANY

Dr. F. Wagner, (ASDEX), Max-Planck-Institut, W. GERMANY

Librarian, Max-Planck-Institut, W. GERMANY

Prof. R.K. Janov, Inst. of Ptrysica, YUGOSLAVIA 

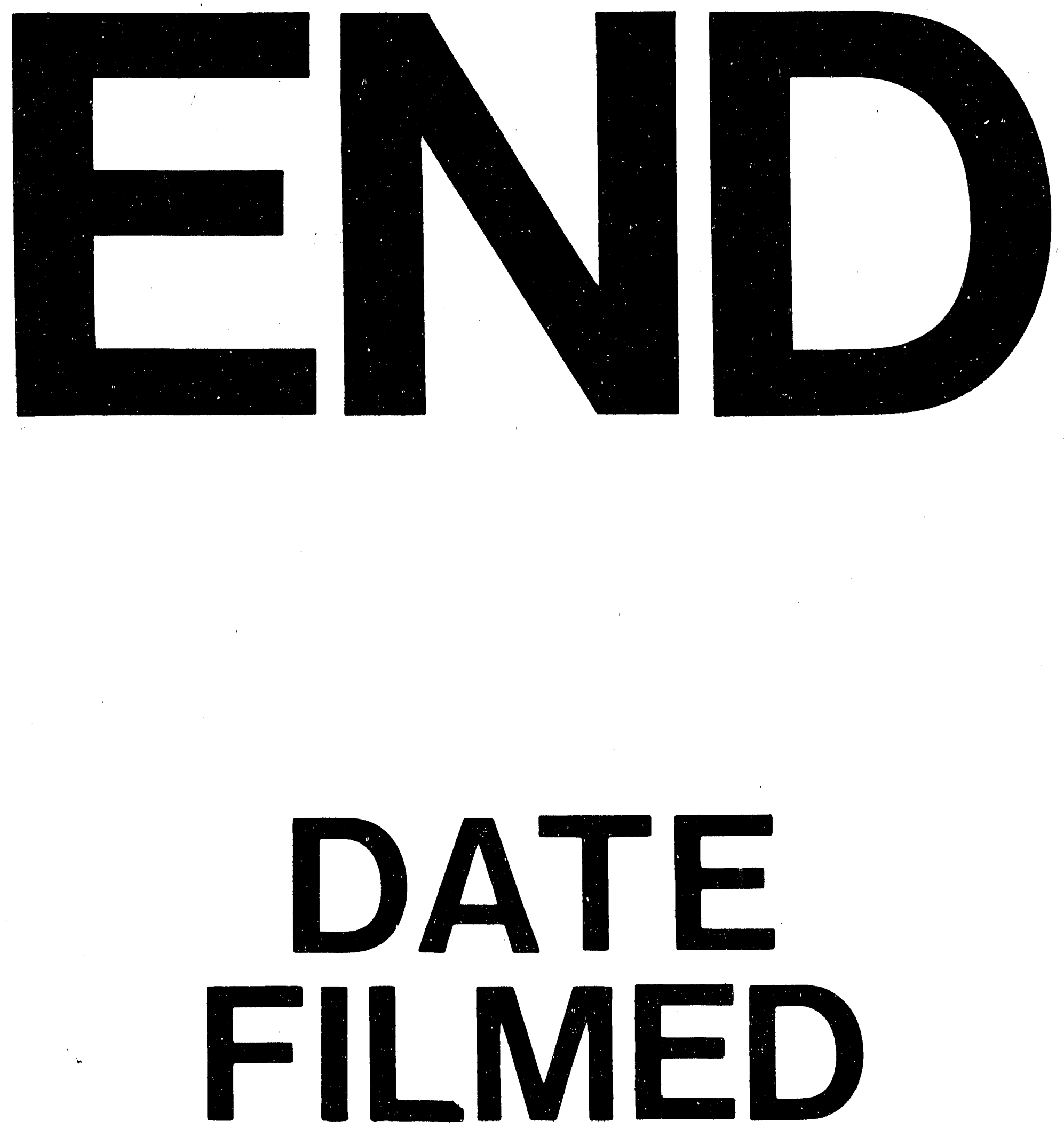

I

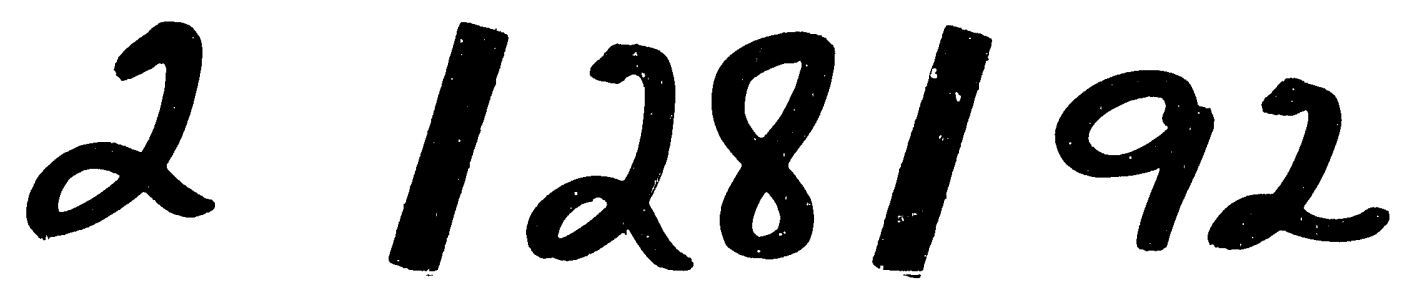




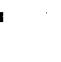

